\title{
Ecophysiological properties of three biological soil crust types and their photoautotrophs from the Succulent Karoo, South Africa
}

\author{
Alexandra Tamm (iD • Jennifer Caesar • Natalie Kunz • \\ Claudia Colesie $\cdot$ Hans Reichenberger $\cdot$ Bettina Weber
}

Received: 16 November 2017 / Accepted: 16 March 2018 / Published online: 27 March 2018

(C) The Author(s) 2018

\begin{abstract}
Background and Aims Biological soil crusts cover about one third of the terrestrial soil surfaces in drylands, fulfilling highly important ecosystem services. Their relevance to global carbon cycling, however, is still under debate.

Methods We utilized $\mathrm{CO}_{2}$ gas exchange measurements to investigate the net photosynthetic response of combined cyanobacteria/cyanolichen-, chlorolichen- and moss-dominated biocrusts and their isolated photoautotrophic components to light, temperature, and water. The results were compared with field studies to evaluate their compatibility.
\end{abstract}

Responsible Editor: Fernando T. Maestre.

Electronic supplementary material The online version of this article (https://doi.org/10.1007/s11104-018-3635-4) contains supplementary material, which is available to authorized users.

A. Tamm $(\bowtie) \cdot$ B. Weber $(\bowtie)$

Multiphase Chemistry Department, Max Planck Institute for Chemistry, Hahn-Meitner-Weg 1, 55128 Mainz, Germany

e-mail: a.tamm@mpic.dee-mail: b.weber@mpic.de

J. Caesar

Department of Agriculture \& Food Sciences, University of Applied Sciences, Neubrandenburg, Germany

N. Kunz $\cdot$ H. Reichenberger

Plant Ecology and Systematics, Biology Department, University of Kaiserslautern, Kaiserslautern, Germany

C. Colesie

Department of Forest Genetics and Plant Physiology, Swedish University of Agricultural Sciences (SLU), Umeå, Sweden
Results Different biocrust types responded similarly, being inhibited by limited and excess water, saturated by increasing light intensities, and having optimum temperatures. Cyanobacteria/cyanolichen-dominated biocrusts reached their water optimum at lowest contents $(0.52-$ $0.78 \mathrm{~mm} \mathrm{H}_{2} \mathrm{O}$ ), were saturated at highest light intensities, and had a comparably high temperature optimum at $37{ }^{\circ} \mathrm{C}$. Chlorolichen-dominated crusts had a medium water optimum $\left(0.75-1.15 \mathrm{~mm} \mathrm{H}_{2} \mathrm{O}\right)$, medium saturating light intensities and a moderate temperature optimum of $22{ }^{\circ} \mathrm{C}$. Moss-dominated biocrusts had the highest water optimum (1.76-2.38 $\left.\mathrm{mm} \mathrm{H}_{2} \mathrm{O}\right)$, lowest saturating light intensities, and a similar temperature optimum at $22{ }^{\circ} \mathrm{C}$. Isolated photoautotrophs responded similar to complete crusts, only isolated moss stems revealed much lower respiration rates compared to complete crusts.

Conclusions In addition to their overall functional similarities, cyanobacteria/cyanolichen-dominated biocrusts appeared to be best adapted to predicted climate change of increasing temperatures and smaller precipitation events, followed by chlorolichen-dominated biocrusts. Mossdominated biocrusts needed by far the largest amounts of water, thus likely being prone to anticipated climate change.

Keywords Biological soil crust $\cdot \mathrm{CO}_{2}$ gas exchange . Photosynthesis $\cdot$ Soil respiration

\section{Introduction}

Biological soil crusts, referred to as biocrusts hereafter, are integral components of drylands on all Earth 
continents, covering an overall area of $\sim 18$ million $\mathrm{km}^{2}$, which corresponds to about $12 \%$ of the global terrestrial surface (Elbert et al. 2012; Rodríguez-Caballero et al. 2018). They consist of communities formed by photoautotrophic cyanobacteria, algae, lichens, and bryophytes growing together with heterotrophic fungi, bacteria, and archaea (Belnap et al. 2016). All biocrust organisms are poikilohydric, as they do not actively regulate their water status, but adapt in a mostly passive way to the water conditions of the surrounding environment (Frahm et al. 2010; Belnap et al. 2016). Under dry conditions they desiccate, becoming physiologically inactive, and upon re-hydration from water, dew, and/or fog, they become active again. High air humidity alone can be utilized by chlorophytes, but not cyanobacteria (Lange 2003a). Biocrusts occur on ground surfaces in drylands throughout the world, and, depending on their prevalent photoautotrophic component, are classified into cyanobacteria-, lichen-, and bryophyte-dominated biocrusts (Büdel et al. 2009; Weber et al. 2015). Lichendominated biocrusts are further separated into the major groups of cyano- and chlorolichen-dominated crusts depending on the photosynthetically active partner in the lichen (i.e., algae or cyanobacteria). In the group of bryophyte-dominated biocrusts, moss- and liverwortdominated crusts are distinguished, depending on the dominating bryophyte phylum (Seppelt et al. 2016).

Biocrusts are highly relevant in dryland ecosystems, as they stabilize and protect the soil against erosion (Lange et al. 1998; Campbell et al. 1989; Beyschlag et al. 2008; Belnap et al. 2016) and influence seed germination and seedling establishment of plants, as grasses and shrubs (Li et al. 2005; Coe et al. 2012). They are hotspots of fungal and bacterial diversity (Maier et al. 2014, 2016, 2018) and have an impact on the composition and structure of the soil (Belnap and Harper 1995; Lange et al. 1998). Biocrusts influence carbon $(\mathrm{C})$ and nitrogen cycling within the soil (Elbert et al. 2012; Reed et al. 2012) and trace gas exchange processes with the atmosphere (Porada et al. 2014; Lenhart et al. 2015; Weber et al. 2015; Meusel et al. 2018).

$\mathrm{CO}_{2}$ release processes from biocrusts to the atmosphere are caused by respiration of living organisms, whereas $\mathrm{CO}_{2}$ uptake is accomplished by photosynthesizing components (Lange et al. 1998). Thus, the overall net photosynthesis (NP) or dark respiration (DR) rate measured on biocrusts represents the sum of all $\mathrm{CO}_{2}$ gas exchange processes occurring during the measurement interval. Measurements of $\mathrm{CO}_{2}$ gas exchange rates give information on the physiological properties of biocrusts with regard to different environmental factors and reveal their overall physiological performance, and the results may be used for calculations of overall $\mathrm{C}$ balances of biocrusts (Lange 2003c; Sancho et al. 2016; Büdel et al. 2018). In all $\mathrm{CO}_{2}$ gas exchange measurements, a potential activity of the substrate alone, as e.g., caused by a dissolution of carbonate, has to be excluded (Sancho et al. 2016).

In the current study, we investigated the $\mathrm{CO}_{2}$ gas exchange properties of cyanobacteria/cyanolichen- and chlorolichen-dominated biocrusts, as well as their isolated photosynthesizing components, and compared the results with those obtained for moss-dominated biocrusts in a previous study (Weber et al. 2012). We conducted factorial analyses in the lab, which covered the full range of temperature, water, and light conditions experienced under natural conditions. Based on these data, we determined the points for water and light compensation, light saturation, and water optimum for NP and DR, to compare the physiological properties of the different biocrust types and their photoautotrophic components. $\mathrm{CO}_{2}$ gas exchange measurements under varying light and water conditions were also conducted in the field to serve as reference data during evaluation of the physiological integrity of biocrust samples after transport and storage. The aims of the current study were to investigate i) maximum rates of photosynthesis within the given range of environmental conditions, ii) the different physiological properties and adaptations of the three main biocrust types with regard to the given environmental conditions and iii) the physiological activity of the photoautotrophic as compared to the heterotrophic biocrust part.

\section{Materials and methods}

Study area

The study was conducted in the vicinity of the BIOTA observatory Soebatsfontein (No. 22, $30.1865^{\circ} \mathrm{S}$, $17.5434^{\circ} \mathrm{E}, 392 \mathrm{~m}$ a.s.1., http://www.biota-africa.org), located within the Succulent Karoo approximately 50 $\mathrm{km}$ south-west of Springbok in the Northern Cape Province, South Africa (Haarmeyer et al. 2010; Kunz et al. 2012; Weber et al. 2012). The Succulent Karoo is a semi-desert with semi-arid climate, where most 
precipitation occurs during the winter months (July and August). In Soebatsfontein, the annual precipitation, including only rainfall in that case, averages about 131 (97 to 175 ) $\mathrm{mm}$, as measured by the BIOTA weather station between the years 2001 and 2009 (Haarmeyer et al. 2010). Since rainfall is very limited in the Succulent Karoo, the relative air humidity and frequent fog events may also promote the high species richness of this area (Haarmeyer et al. 2010). The mean air temperature is $19.4{ }^{\circ} \mathrm{C}$ and varies between $\sim 42.5^{\circ} \mathrm{C}$ in February and $\sim 3.5^{\circ} \mathrm{C}$ in July (Haarmeyer et al. 2010).

The topography and geology of this area is characterized by broad valleys and undulating hills often made up by granite inselbergs (up to $400 \mathrm{~m}$ a.s.l.). The region is strongly influenced by fossil termite mounds (socalled heuweltjies), which cause a high variability of soil parameters (Dojani et al. 2014). Heuweltjie soils are characterized by finer texture, higher $\mathrm{pH}$-values and higher concentrations of $\mathrm{C}, \mathrm{N}$, and $\mathrm{P}$ compared to the surrounding soils (Kunz et al. 2012). Soils are generally shallow with varying underlying bedrock of granite and quartz and soil texture ranges from sandy to silty loam represented by three dominant soil types, namely Leptosols, Durisols, and Cambisols (Haarmeyer et al. 2010). The area is situated within the Succulent Karoo biome, which is known for a unique flora of succulent plants and very high plant diversity (Van Jaarsveld 1987). The perennial dwarf shrub vegetation, with Aizoaceae and Asteraceae as the dominating families, covers about $23 \%$ of the land surface (Haarmeyer et al. 2010). The land is utilized by means of small stock farming, and in regions with higher grazing pressure perennial species tend to be lost causing a shift to annual plants and geophytes (Kunz et al. 2012; Haarmeyer et al. 2010).

The Soebatsfontein region is characterized by a dense coverage of biological soil crusts, covering up to one third of the landscape and reaching an overall biomass value of about $480 \mathrm{~g} \mathrm{ha}^{-1}$ of chlorophyll $\mathrm{a}+\mathrm{b}$ at the landscape scale (Rodríguez-Caballero et al. 2017). Within the study area, cyanobacteriadominated biocrusts reach the highest cover values with a minor presence of lichen- and bryophytedominated biocrusts (Bettina Weber, personal observation). Moss-dominated biocrusts mainly occur below shrubs, whereas cyanobacteria/cyanolichen- and lichen-dominated biocrusts grow both beneath vegetation and in the open plant interspaces (Dojani et al. 2011; Weber et al. 2012).
Sampling and storage

Two different types of biocrusts and bare soil samples were collected for this study. The first biocrust type was dominated by cyanobacteria and cyanolichens (hereafter called cyanobacteria/cyanolichen-dominated biocrust), as it comprised not only cyanobacteria, with genera such as Chroococcidiopsis, Pseudanabaena, Phormidium, Leptolyngbya, Microcoleus, and Nostoc (Büdel et al. 2009), but also a $30-80 \%$ cover of the gelatinous cyanolichen Collema coccophorum Tuck. (Fig. 1a, b). Chlorolichen-dominated biocrusts (the second type) were dominated by Psora crenata (Taylor) Reinke and P. decipiens (Hedwig) Hoffm. (Fig. 1c, d). $P$. crenata is indeed phylogenetically and morphologically closely related to $P$. decipiens (Eldridge and Ferris 1999; Belnap et al. 2001; Büdel et al. 2013), but is separated by the presence of larger squamules with a prominent central depression and the presence of the norstictic acid (Rosentreter et al. 2007; Leavitt et al. 2018). P. decipiens has been shown to play a crucial role in biocrust plasticity (Colesie et al. 2017) and acclimation potential (Williams et al. 2017). A mossdominated biocrust (the third type), dominated by the species Ceratodon purpureus (Hedw.) Brid (Fig. 1e, f) also occurs at the site and was previously investigated (Weber et al. 2012). For our laboratory measurements, cyanobacteria/cyanolichen- and chlorolichendominated biocrusts were collected in October 2010 (after the rainy season).

For sample collection, a petri dish (lined with some pieces of soft cellulose tissue) was pressed upside down into biocrusted soil to collect the upper $0.5-1 \mathrm{~cm}$. A trowel was used to lift the soil together with the petri dish. The sample was then turned upside down and airdried under ambient conditions. After drying, the petri dishes were closed with the corresponding lids and sealed with Parafilm (Brand GmbH, Wertheim, Germany). The samples were transported to the University of Kaiserslautern, where they were stored in a freezer at $-20{ }^{\circ} \mathrm{C}$ until measurements in winter 2011/2012 (chlorolichen-dominated biocrusts) and in spring 2013 (cyanobacteria/cyanolichen-dominated biocrusts). Previous measurements have shown that dry biocrusts could outlast under these conditions without harm (Weber et al. 2012). During a field campaign in November 2013, samples were taken in the same way as described above, but then placed in metal baskets for field measurements. 
Fig. 1 Different types of biocrusts. Pictures present closeup views of the respective biocrust types taken with a camera mounted on a stereomicroscope. These are cyanobacteria/ cyanolichen-dominated biocrusts (a, b); chlorolichen-dominated biocrusts with Psora crenata (Taylor) Reinke and Psora decipiens (Hedwig) Hoffm. as dominating species (c, d); mossdominated biocrusts with Ceratodon purpureus (Hedw.) Brid as dominating species (e, f). Pictures a, $\mathbf{c}$ and $\mathbf{e}$ show biocrusts in a dry and pictures $\mathbf{b}, \mathbf{d}$ and $\mathbf{f}$ in a wet status
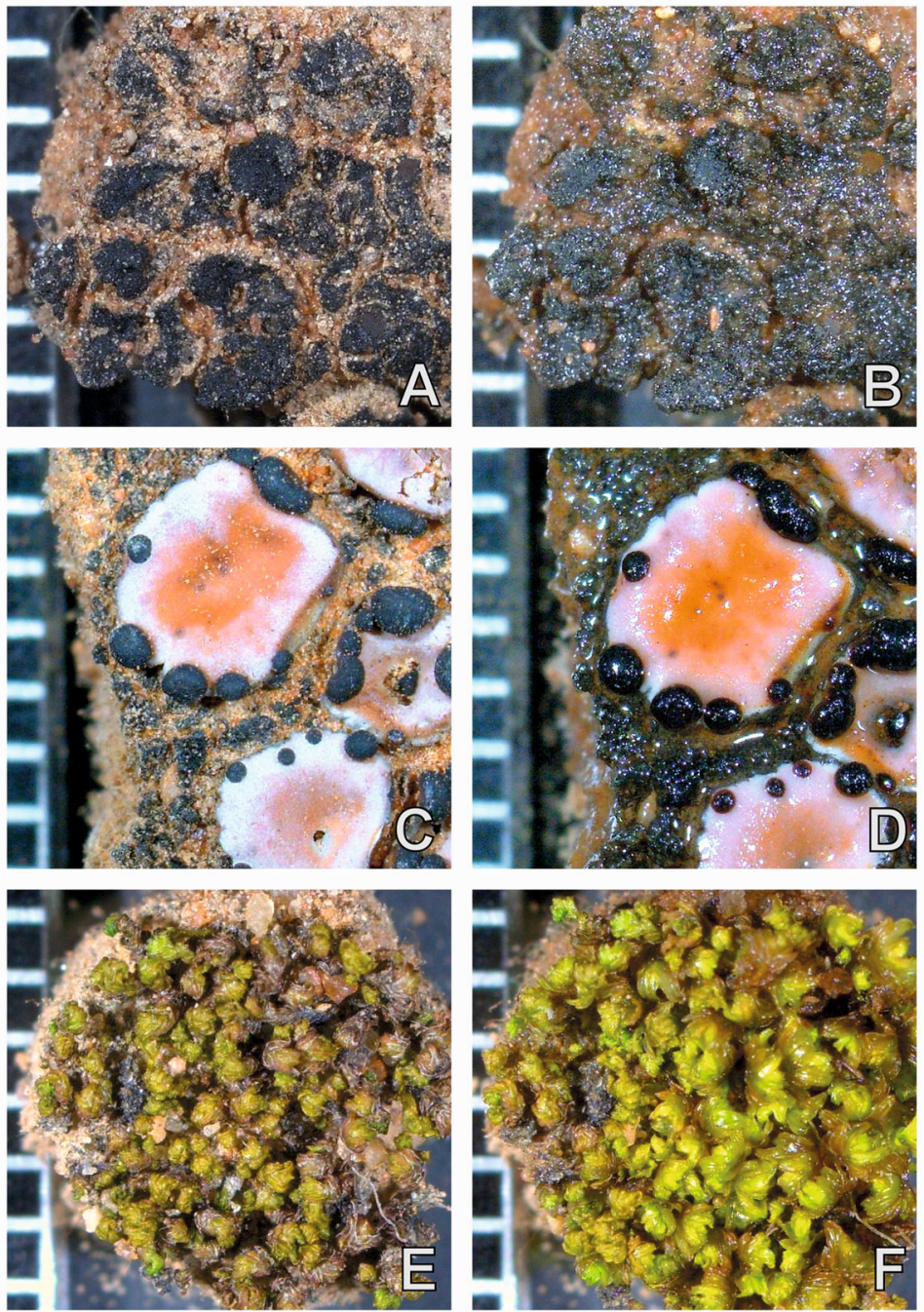

$\mathrm{CO}_{2}$ gas exchange measurements under controlled conditions in the lab

Prior to measurements, the petri dishes were defrosted at ambient temperature. Samples of appropriate size were placed into stainless steel baskets $(3.5 \times 2.5 \mathrm{~cm})$ and fixed with gas-inert stainless steel wire under a stereo microscope (Stemi DV4, Zeiss, Oberkochen, Germany). Subsequently, the samples were kept for at least $48 \mathrm{~h}$ in a climate chamber at $17-22{ }^{\circ} \mathrm{C}$ and about $50-100 \mu \mathrm{mol} \mathrm{m}{ }^{-2} \mathrm{~s}^{-1}$ PPFD (photosynthetic photon flux density) under a light-dark regime of 12:12 h. Samples were sprayed once a day with distilled water to activate them.

The $\mathrm{CO}_{2}$ gas exchange measurements were performed under controlled laboratory conditions, utilizing a portable gas exchange system (GFS 3000, Walz
$\mathrm{GmbH}$, Effeltrich, Germany) with an LED light source providing $90 \%$ red and $10 \%$ blue light (3040-L, Walz $\mathrm{GmbH}$, Effeltrich, Germany). The complete measurement setup was placed in a climate chamber to assure stable measurement conditions and to avoid condensation within the cuvette, tubes, or measuring instruments. The measurement setup was equipped with a humidification bottle and a subsequent temperature-controlled measuring gas cooler (MGK-1, Walz GmbH, Effeltrich, Germany) so air humidity could be controlled and excess water removed from the gas after its passage through the cuvette. Prior to investigation of the biocrust samples, $\mathrm{CO}_{2}$ gas exchange of bare soil samples collected within the study region was measured and it was verified that bare soil was inert with regard to $\mathrm{CO}_{2}$ exchange (data not shown). 
We first measured water response curves at $17^{\circ} \mathrm{C}$ and $500 \mu \mathrm{mol} \mathrm{m}{ }^{-2} \mathrm{~s}^{-1}$ PPFD. For that, the samples were wetted to full water holding capacity and NP recorded until the samples were well beyond maximum NP levels or completely dry again. Subsequently, combined light and water response curves were performed at seven different temperatures $\left(7,12,17,22,27,32\right.$ and $\left.37^{\circ} \mathrm{C}\right)$. At each temperature step, the samples were wetted to full water holding capacity and light cycles with increasing light intensities $(0,15,25,50,100,150,300,500,750$, 1000, 1250, $1500 \mu \mathrm{mol} \mathrm{m} \mathrm{m}^{-2} \mathrm{~s}^{-1}$ PPFD) were repeated until full desiccation. Each light cycle took about $30 \mathrm{~min}$, i.e. $2.5 \mathrm{~min}$ per light intensity. Before and after each light cycle, the sample was removed from the cuvette and weighed to determine the water content. Prior to placing the sample back into the chamber, a short (3-5 min) zero value was determined for reference purposes. The measurements were conducted at $80-90 \%$ relative air humidity, an airflow of $600 \mu \mathrm{mol} \mathrm{s}^{-1}$ and a fixed $\mathrm{CO}_{2}$ concentration of $380 \mathrm{ppm}$.

After finalization of the water and light response curves, a second water response curve at $17{ }^{\circ} \mathrm{C}$ and $500 \mu \mathrm{mol} \mathrm{m}{ }^{-2} \mathrm{~s}^{-1}$ PPFD was performed to evaluate the physiological stability of the samples. In contrast to the moss-dominated biocrusts, which had previously been measured (Weber et al. 2012), both the cyanobacteria/cyanolichen- and chlorolichendominated biocrusts were stable throughout the measurements. Subsequently, the biocrust fraction below the photoautotrophic organisms was removed with the help of tweezers and distilled water, carefully avoiding a damage of the rhizines and thalli of the samples. In contrast to cyanobacteria/cyanolichen- and chlorolichen-dominated the moss-dominated biocrusts were carefully separated into moss stems/ photoautotrophic components and remaining substrate, which was used for further measurements as described by Weber et al. (2012). For subsequent measurements of the photoautotrophic components, the metal baskets were adjusted to guarantee an equal distance between the light source and the organisms. Then, the measurements described above were conducted with the photoautotrophic biocrust fraction, although an undetermined amount of fungal and bacterial components would still be present. For each biocrust type, three replicates were characterized, one after the other, according to this protocol.

The surface area of the samples was determined using digital photos of the samples and the software
ImageJ (National Institutes of Health, Bethesda, MD, USA). After finalization of the measurements, the biocrust fractions (photoautotrophic components and remaining substrate) were dried in an oven at $60{ }^{\circ} \mathrm{C}$ and total dry weight was determined. Chlorophyll extraction was performed according to the method described by Caesar et al. (2018; see supplement for methods description), but without intermediate shaking, using Dimethylsulfoxide (DMSO) plus a spatula tip of $\mathrm{CaCO}_{3}$ to avoid acidification and concomitant phaeophytinization of chlorophyll (Ronen and Galun 1984). After spectrophotometry, the chlorophyll $\left(\mathrm{Chl}_{\mathrm{a}}\right)$ content was calculated according to Arnon et al. 1974, whereas chlorophyll $\mathrm{a}_{\mathrm{b}}\left(\mathrm{Ch}_{\mathrm{a}+\mathrm{b}}\right)$ was determined according to Lange (pers. comm.; Weber et al. 2013; Caesar et al. 2017).

$\mathrm{CO}_{2}$ gas exchange measurements in the field

$\mathrm{CO}_{2}$ gas exchange measurements in the field were performed at night, since daytime temperatures were too high and fluctuations were too large to allow measurements at a fixed temperature. For measurements with the portable gas exchange system (GFS 3000 equipped with LED light source (3040-L); Walz GmbH, Effeltrich, Germany), the samples were moistened and light cycles with increasing light intensities $(0,100,500,1000$ and $1500 \mu \mathrm{mol} \mathrm{m} \mathrm{m}^{-2} \mathrm{~s}^{-1}$ PPFD) were repeated until the samples were dry. . In sum, we investigated five cyanobacteria/cyanolichen, five chlorolichen- and six moss-dominated biocrusts (same biocrust types as those characterized in laboratory measurements of this and a previous study; Weber et al. 2012).

Data analysis

Since the dry weight of all samples was heavily dependent on the amount of soil beneath the biocrust, the response curves were related to $\mathrm{mm}$ rainfall equivalent (i.e. amount of water per surface area); however, these can easily be converted into water content based on the dry weight or chlorophyll content (Table S1). Optimum water contents were defined as the span over which at least $90 \%$ of the maximum net photosynthesis rate was reached. Light response curves represent light cycles at optimum water content measured at each temperature. Using water response curves, the water compensationpoint, defined as the water contents at which photosynthetic $\mathrm{CO}_{2}$ uptake is compensated by respiration, were 
determined for all biocrust types plus photoautotrophic components (Lange 1980). Light saturation was calculated as the light intensity where $95 \%$ of maximum NP were reached (Lange et al. 1998). Subsequently, for all samples of each crust type, mean values for each temperature step were determined and the obtained data were smoothened using the Smith function (Smith 1937, 1938) as described by Lange et al. (1991). Three-dimensional graphs were designed using the software SigmaPlot (SigmaPlot 10.0, Systat Software Inc., San Jose (USA), Bangalore (India)). Average values of different replicates are always shown as mean values \pm standard deviation (SD).

For statistical analyses (OriginPro 2015 64-Bit, OriginLab Corporation, Northampton, Massachusetts, USA), all data were first tested for normality (ShapiroWilk test/Anderson-Darling test) and homogeneity of variance (Levene's test/Brown-Forsythe test). Absolute values of light compensation points, NP/DR ratio, and DR (at optimum water content, all temperatures) needed transformation via a natural logarithm ( $\mathrm{ln}$ ), reciprocal, and root transformation, respectively, to obtain normally distributed data. A T-test was performed to compare water compensation points of biocrusts and photoautotrophic components. A one-way ANOVA with a Bonferroni post hoc test was conducted to compare water compensation points and maximum NP values of the different biocrust types $\left(500 \mu \mathrm{mol} \mathrm{m}{ }^{-2} \mathrm{~s}^{-1} \mathrm{PPFD}, 17^{\circ} \mathrm{C}\right.$, optimum water content), as well as maximum NP values of field vs. laboratory measurements at $22{ }^{\circ} \mathrm{C}$ (Table S2, S4, S5). The effect of temperature and biocrust type on light compensation points, NP/DR ratio, maximum NP and DR at maximum NP (at optimum water content, all temperatures, and optimum temperature) was analyzed using a two-way ANOVA with a Bonferroni post hoc test (Table S3). For all tests a significance level of $p \leq 0.05$ was chosen.

Data availability All data used in this paper are available from the corresponding authors upon request.

\section{Results}

Response of $\mathrm{CO}_{2}$ gas exchange to changing water contents

Net photosynthesis of all three biocrust types (complete biocrusts) and their respective photoautotrophic components responded with optimum curves that decreased at the lower and higher water contents (Fig. 2). Optimum water contents for moss-dominated biocrusts were $\sim 3.5$ and $\sim 2$ times higher than those of cyanobacteria/ cyanolichen- and chlorolichen-dominated biocrusts, respectively (cyanobacteria/cyanolichen: $0.52-0.78 \mathrm{~mm}$ $\mathrm{H}_{2} \mathrm{O}$; chlorolichen: $0.75-1.15 \mathrm{~mm} \mathrm{H}_{2} \mathrm{O}$; moss: 1.76$2.38 \mathrm{~mm} \mathrm{H}_{2} \mathrm{O}$; Table 1). While moss stems showed similar optimum water contents compared to the complete crust (1.77-2.28 $\mathrm{mm} \mathrm{H}_{2} \mathrm{O}$ ), the photoautotrophic components from the cyanobacteria/cyanolichen- and chlorolichen-dominated biocrusts had lower optimum water contents $\left(0.28-0.55 \mathrm{~mm} \mathrm{H}_{2} \mathrm{O}\right.$ and $0.34-$ $0.73 \mathrm{~mm} \mathrm{H}_{2} \mathrm{O}$, respectively; Table 1). Maximum NP rates at $17{ }^{\circ} \mathrm{C}$ and $500 \mu \mathrm{mol} \mathrm{m} \mathrm{s}^{-2}$ PPFD were significantly lower for cyanobacteria/cyanolichen- $(0.7$ $\left.\pm 0.2 \mu \mathrm{mol} \mathrm{CO} \mathrm{CO}_{2}^{-2} \mathrm{~s}^{-1}\right)$, compared to moss- $(2.3 \pm$ $0.6 \mu \mathrm{mol} \mathrm{CO}_{2} \mathrm{~m}^{-2} \mathrm{~s}^{-1}$ ) and chlorolichen-dominated biocrusts $\left(3.0 \pm 0.2 \mu \mathrm{mol} \mathrm{CO} \mathrm{CO}^{-2} \mathrm{~s}^{-1}\right.$; Fig. 2a, c, e, Table 1, Table S2). The photoautotrophic components of cyanobacteria/cyanolichen- and chlorolichendominated biocrusts reached similar maximum NP values as the complete biocrusts (i.e., $1.0 \pm 0.2 \mu \mathrm{mol}$ $\mathrm{CO}_{2} \mathrm{~m}^{-2} \mathrm{~s}^{-1}$ and $3.1 \pm 0.0 \mu \mathrm{mol} \mathrm{CO} \mathrm{Cm}^{-2} \mathrm{~s}^{-1}$, respectively), whereas values of isolated moss stems were significantly higher compared to moss-dominated biocrusts $\left(3.4 \pm 0.5 \mu \mathrm{mol} \mathrm{CO} \mathrm{CO}^{-2} \mathrm{~s}^{-1}\right.$; Fig. $2 \mathrm{~b}, \mathrm{~d}, \mathrm{f}$, Table 1, Table S2). In contrast, water compensation points showed no significant differences between biocrust types and photoautotrophic components, although complete biocrusts reached the compensation point at slightly higher water contents compared to the photoautotrophic components (Fig. 2, Table 1, Table S2). Only after pooling the data, significant differences between biocrusts and photoautotrophic components $(\mathrm{DF}=16$; $\mathrm{t}$-value $=2.71 ; p$-value $=0.016)$ were observed (Fig. 2, Table 1).

Response of $\mathrm{CO}_{2}$ gas exchange to changing light and temperature

All types of complete biocrusts as well as their isolated photoautotrophs responded with saturation curves upon increasing photosynthetic PPFD. With increasing temperature, saturating PPFD increased for both complete biocrusts and isolated photoautotrophs (Table 2). In general, saturating light intensities were highest for cyanobacteria/cyanolichen-dominated biocrusts (752 to $1301 \mu \mathrm{mol} \mathrm{m} \mathrm{m}^{-2} \mathrm{~s}^{-1}$ PPFD), being followed by chlorolichen- and moss-dominated biocrusts (723 to 

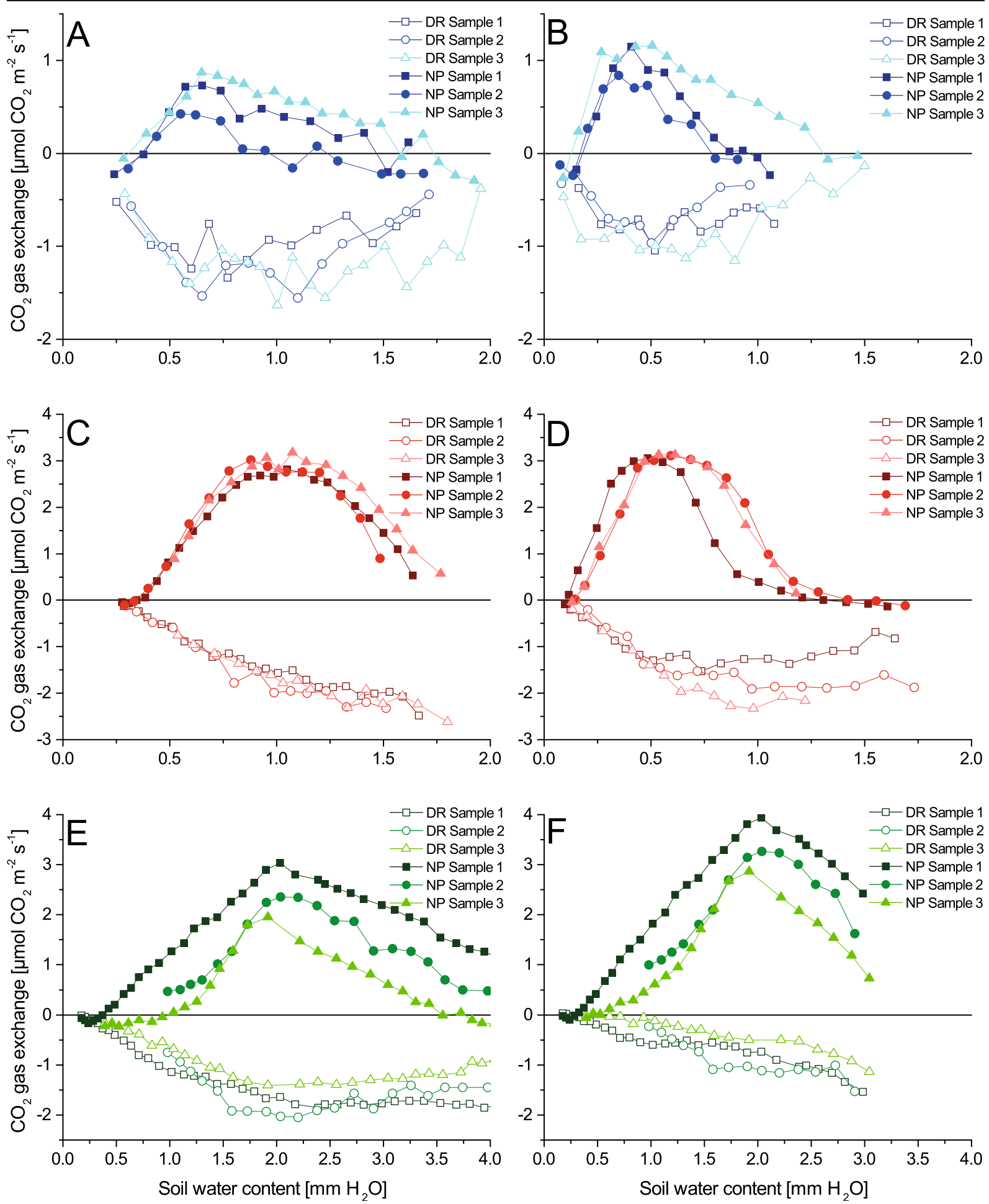

Fig. 2 Response of net photosynthesis (NP; closed symbols) and dark respiration (DR; open symbols) to thallus water content (calculated as mm-precipitation equivalent) of different biocrust types and their isolated photoautotrophic components $(n=3)$. These are cyanobacteria/cyanolichen-dominated biocrusts (a), isolated cyanobacteria/cyanolichens (b), chlorolichen-dominated

biocrusts (c), isolated chlorolichens (d), moss-dominated biocrusts (e), and isolated moss stems (f). Complete desiccation cycles (saturated to air dry) were conducted at $500 \mu \mathrm{mol} \mathrm{m-2} \mathrm{s-1} \mathrm{PPFD}$ and in the dark, at $17^{\circ} \mathrm{C}$, and $380 \mathrm{ppm}$. Data on moss-dominated biocrusts and moss stems are taken from Weber et al. (2012) 
Table 1 Cardinal points of $\mathrm{CO}_{2}$ gas exchange water/light response curves obtained for cyanobacteria/cyanolichen-, chlorolichen-, and moss-dominated biocrusts and their isolated photoautotrophs

\begin{tabular}{|c|c|c|c|c|}
\hline & Sample 1 & Sample 2 & Sample 3 & Mean value $\pm \mathrm{SD}$ \\
\hline \multicolumn{5}{|l|}{ Water compensation point at $17^{\circ} \mathrm{C}\left[\mathrm{mm} \mathrm{H}_{2} \mathrm{O}\right]$} \\
\hline Cyanobacteria/cyanolichen-dominated crust & 0.39 & 0.38 & 0.31 & $0.36 \pm 0.04$ \\
\hline Cyanobacteria/cyanolichens & 0.18 & 0.16 & 0.13 & $0.16 \pm 0.03$ \\
\hline Chlorolichen-dominated crust & 0.34 & 0.34 & 0.20 & $0.29 \pm 0.08$ \\
\hline Chlorolichens & 0.11 & 0.14 & 0.12 & $0.12 \pm 0.02$ \\
\hline Moss-dominated crust & 0.36 & 0.82 & 0.98 & $0.72 \pm 0.32$ \\
\hline Moss stems & 0.28 & 0.64 & 0.42 & $0.45 \pm 0.18$ \\
\hline \multicolumn{5}{|l|}{ Optimum water contents at $17^{\circ} \mathrm{C}\left[\mathrm{mm} \mathrm{H}_{2} \mathrm{O}\right]$} \\
\hline Cyanobacteria/cyanolichen-dominated crust & $0.61-0.69$ & $0.52-0.71$ & $0.70-0.78$ & $0.52-0.78$ \\
\hline Cyanobacteria/cyanolichens & $0.28-0.45$ & $0.31-0.38$ & $0.40-0.55$ & $0.28-0.55$ \\
\hline Chlorolichen-dominated crust & $0.78-1.15$ & $0.75-1.09$ & $0.79-1.13$ & $0.75-1.15$ \\
\hline Chlorolichens & $0.34-0.51$ & $0.48-0.73$ & $0.50-0.65$ & $0.34-0.73$ \\
\hline \multicolumn{5}{|l|}{ Optimum water contents at optimum temperatures $\left[\mathrm{mm} \mathrm{H}_{2} \mathrm{O}\right]$} \\
\hline Cyanobacteria/cyanolichen-dominated crust $\left(37^{\circ} \mathrm{C}\right)$ & $0.58-0.69$ & $0.75-0.87$ & $0.92-1.38$ & $0.58-1.38$ \\
\hline Cyanobacteria/cyanolichens $\left(27^{\circ} \mathrm{C}\right)$ & $0.29-0.46$ & $0.30-0.39$ & $0.39-0.59$ & $0.29-0.59$ \\
\hline Chlorolichen-dominated crust $\left(22^{\circ} \mathrm{C}\right)$ & $0.67-1.13$ & $0.74-1.09$ & $0.78-1.10$ & $0.67-1.13$ \\
\hline Chlorolichens $\left(22^{\circ} \mathrm{C}\right)$ & $0.32-0.47$ & $0.49-0.69$ & $0.48-0.72$ & $0.32-0.72$ \\
\hline Moss-dominated crust $\left(22^{\circ} \mathrm{C}\right)$ & & & & $1.76-2.38$ \\
\hline Moss stems $\left(22^{\circ} \mathrm{C}\right)$ & & & & $1.77-2.28$ \\
\hline \multicolumn{5}{|l|}{ Maximum NP rate at $17^{\circ} \mathrm{C}\left[\mu \mathrm{mol} \mathrm{CO} \mathrm{m}^{-2} \mathrm{~s}^{-1}\right]$} \\
\hline Cyanobacteria/cyanolichen-dominated crust & 0.7 & 0.4 & 0.9 & $0.7 \pm 0.2$ \\
\hline Cyanobacteria/cyanolichens & 1.1 & 0.8 & 1.2 & $1.0 \pm 0.2$ \\
\hline Chlorolichen-dominated crust & 2.8 & 3.0 & 3.2 & $3.0 \pm 0.2$ \\
\hline Chlorolichens & 3.1 & 3.1 & 3.1 & $3.1 \pm 0.0$ \\
\hline Moss-dominated crust & 2.9 & 2.3 & 1.7 & $2.3 \pm 0.6$ \\
\hline Moss stems & 3.9 & 3.3 & 2.9 & $3.4 \pm 0.5$ \\
\hline
\end{tabular}

Data represent water compensation points at $17^{\circ} \mathrm{C}\left[\mathrm{mm} \mathrm{H}_{2} \mathrm{O}\right]$, optimum water contents at $17{ }^{\circ} \mathrm{C}\left[\mathrm{mm} \mathrm{H}_{2} \mathrm{O}\right]$, optimum water contents at optimum temperature $\left[\mathrm{mm} \mathrm{H}_{2} \mathrm{O}\right]$, and maximum $\mathrm{NP}$ rates at $17^{\circ} \mathrm{C}\left[\mu \mathrm{mol} \mathrm{CO} \mathrm{Cm}^{-2} \mathrm{~s}^{-1}\right]$. Water compensation points, maximum $\mathrm{NP}$ rates, and optimum water contents at $17{ }^{\circ} \mathrm{C}$ were determined using water response curves $\left(500 \mu \mathrm{mol} \mathrm{m}^{-2} \mathrm{~s}^{-1}\right.$ PPFD); optimum water contents were determined across light cycles $\left(0-1500 \mu \mathrm{mol} \mathrm{m} \mathrm{m}^{-2} \mathrm{PPFD}\right)$. All measurements were performed at $380 \mathrm{ppm} \mathrm{CO}_{2}$. Values obtained for the individual samples and mean values \pm SD are shown $(n=3)$. Data on moss-dominated biocrusts and moss stems are taken from Weber et al. (2012)

1249 and 468 to $1135 \mu \mathrm{mol} \mathrm{m}{ }^{-2} \mathrm{~s}^{-1}$ PPFD, respectively; Fig. 3, Table 2). In addition, light compensation points were higher at increasing temperatures, being significantly higher for cyanobacteria/cyanolichen- (123 to $314 \mu \mathrm{mol} \mathrm{m} \mathrm{m}^{-2} \mathrm{~s}^{-1}$ PPFD) compared to chlorolichenand moss-dominated biocrusts (61 to 362 and 32 to $319 \mu \mathrm{mol} \mathrm{m}{ }^{-2} \mathrm{~s}^{-1}$ PPFD, respectively; Fig. 3, Table 2, Table S3). In contrast to other biocrust types, only mossdominated biocrusts had significantly higher light compensation points than isolated moss stems (Fig. 3, Table 2, Table S3).
Cyanobacteria/cyanolichen-dominated biocrusts and their isolated photoautotrophs reached highest NP rates at 37 and $27{ }^{\circ} \mathrm{C}$ with values of 2.7 and $2.3 \mu \mathrm{mol} \mathrm{CO}$ $\mathrm{m}^{-2} \mathrm{~s}^{-1}$, respectively (Fig. 3, Table 2). Comparatively, chlorolichen- and moss-dominated biocrusts reached maxima at lower temperature $\left(22{ }^{\circ} \mathrm{C} ; 3.9\right.$ and $2.8 \mu \mathrm{mol} \mathrm{CO} \mathrm{CO}^{-2} \mathrm{~s}^{-1}$, respectively; Fig. 3, Table 2). At optimum temperatures, the isolated photoautotrophs reached similar NP maxima as the complete crusts in the cyanobacteria/cyanolichen- and chlorolichendominated biocrusts, whereas the isolated moss stems 
Table 2 Cardinal points of combined light and water $\mathrm{CO}_{2}$ gas exchange response curves of cyanobacteria/cyanolichen-, chlorolichen-, and moss-dominated biocrusts and their isolated photoautotrophs under optimum water conditions

\begin{tabular}{|c|c|c|c|c|c|c|c|}
\hline & \multicolumn{7}{|c|}{ Temperature $\left[{ }^{\circ} \mathrm{C}\right]$} \\
\hline & 7 & 12 & 17 & 22 & 27 & 32 & 37 \\
\hline \multicolumn{8}{|l|}{ Light compensation point $\left[\mu \mathrm{mol} \mathrm{m} \mathrm{m}^{-2} \mathrm{~s}^{-1} \mathrm{PPFD}\right]$} \\
\hline Cyanobacteria/cyanolichen- dominated crust & $123 \pm 19$ & $169 \pm 21$ & $209 \pm 47$ & $246 \pm 6$ & $282 \pm 38$ & $314 \pm 44$ & $309 \pm 54$ \\
\hline Cyanobacteria/cyanolichens & $120 \pm 45$ & $134 \pm 11$ & $136 \pm 23$ & $180 \pm 51$ & $213 \pm 33$ & $234 \pm 38$ & $272 \pm 23$ \\
\hline Chlorolichen-dominated crust & $61 \pm 8$ & $66 \pm 6$ & $95 \pm 9$ & $111 \pm 2$ & $174 \pm 19$ & $225 \pm 32$ & $362 \pm 34$ \\
\hline Chlorolichens & $53 \pm 8$ & $62 \pm 6$ & $75 \pm 8$ & $98 \pm 12$ & $139 \pm 16$ & $202 \pm 23$ & $361 \pm 29$ \\
\hline Moss-dominated crust & $32 \pm 2$ & $61 \pm 18$ & $80 \pm 12$ & $112 \pm 38$ & $143 \pm 34$ & $255 \pm 93$ & $319 \pm 34$ \\
\hline Moss stems & $21 \pm 4$ & $30 \pm 19$ & $46 \pm 24$ & $38 \pm 18$ & $52 \pm 17$ & $76 \pm 34$ & $95 \pm 2$ \\
\hline \multicolumn{8}{|l|}{ Saturating irradiance $\left[\mu \mathrm{mol} \mathrm{m} \mathrm{m}^{-2} \mathrm{~s}^{-1} \mathrm{PPFD}\right]$} \\
\hline Cyanobacteria/cyanolichen- dominated crust & $752 \pm 119$ & $907 \pm 16$ & $1159 \pm 78$ & $1212 \pm 24$ & $1283 \pm 59$ & $1301 \pm 32$ & $1295 \pm 47$ \\
\hline Cyanobacteria/cyanolichens & $702 \pm 187$ & $815 \pm 46$ & $983 \pm 103$ & $1010 \pm 113$ & $1155 \pm 48$ & $1202 \pm 16$ & $1224 \pm 16$ \\
\hline Chlorolichen-dominated crust & $723 \pm 58$ & $797 \pm 13$ & $869 \pm 14$ & $929 \pm 19$ & $1030 \pm 29$ & $1132 \pm 64$ & $1249 \pm 28$ \\
\hline Chlorolichens & $731 \pm 38$ & $761 \pm 33$ & $793 \pm 43$ & $861 \pm 38$ & $962 \pm 49$ & $1078 \pm 57$ & $1227 \pm 37$ \\
\hline Moss-dominated crust & $468 \pm 35$ & $569 \pm 126$ & $709 \pm 95$ & $814 \pm 204$ & $881 \pm 146$ & $1073 \pm 112$ & $1135 \pm 26$ \\
\hline Moss stems & $446 \pm 40$ & $519 \pm 139$ & $651 \pm 118$ & $702 \pm 209$ & $780 \pm 89$ & $823 \pm 73$ & $760 \pm 91$ \\
\hline \multicolumn{8}{|l|}{ Maximum NP rates $\left[\mu \mathrm{mol} \mathrm{CO} \mathrm{Cm}^{-2} \mathrm{~s}^{-1}\right]$} \\
\hline Cyanobacteria/cyanolichen- dominated crust & $0.5 \pm 0.1$ & $0.8 \pm 0.3$ & $1.4 \pm 0.2$ & $1.7 \pm 0.1$ & $2.1 \pm 0.2$ & $2.5 \pm 0.3$ & $2.7 \pm 0.4$ \\
\hline Cyanobacteria/cyanolichens & $0.4 \pm 0.2$ & $0.8 \pm 0.2$ & $1.6 \pm 0.3$ & $1.6 \pm 0.3$ & $2.3 \pm 0.5$ & $2.2 \pm 0.2$ & $2.2 \pm 0.0$ \\
\hline Chlorolichen-dominated crust & $2.5 \pm 0.2$ & $3.5 \pm 0.2$ & $3.8 \pm 0.2$ & $3.9 \pm 0.1$ & $3.4 \pm 0.1$ & $2.7 \pm 0.2$ & $1.6 \pm 0.1$ \\
\hline Chlorolichens & $2.5 \pm 0.3$ & $3.4 \pm 0.2$ & $3.9 \pm 0.2$ & $4.0 \pm 0.1$ & $3.5 \pm 0.3$ & $3.0 \pm 0.7$ & $1.5 \pm 0.2$ \\
\hline Moss-dominated crust & $2.4 \pm 0.9$ & $2.3 \pm 0.8$ & $2.2 \pm 0.5$ & $2.8 \pm 0.5$ & $2.6 \pm 0.6$ & $1.6 \pm 0.8$ & $0.9 \pm 0.6$ \\
\hline Moss stems & $2.6 \pm 0.9$ & $2.9 \pm 0.8$ & $3.1 \pm 0.5$ & $4.0 \pm 0.5$ & $4.0 \pm 0.5$ & $3.1 \pm 0.9$ & $2.7 \pm 0.6$ \\
\hline \multicolumn{8}{|l|}{ DR rates at maximum $\mathrm{NP}\left[\mu \mathrm{mol} \mathrm{CO} \mathrm{Cm}^{-2} \mathrm{~s}^{-1}\right]$} \\
\hline Cyanobacteria/cyanolichen- dominated crust & $-0.7 \pm 0.1$ & $-1.1 \pm 0.2$ & $-1.0 \pm 0.3$ & $-1.4 \pm 0.1$ & $-1.5 \pm 0.0$ & $-2.1 \pm 0.2$ & $-2.2 \pm 0.1$ \\
\hline Cyanobacteria/cyanolichens & $-0.5 \pm 0.2$ & $-0.9 \pm 0.2$ & $-1.0 \pm 0.2$ & $-1.6 \pm 0.2$ & $-1.7 \pm 0.4$ & $-1.8 \pm 0.6$ & $-2.2 \pm 0.6$ \\
\hline Chlorolichen-dominated crust & $-0.8 \pm 0.1$ & $-1.2 \pm 0.0$ & $-1.7 \pm 0.2$ & $-2.0 \pm 0.2$ & $-2.5 \pm 0.2$ & $-2.7 \pm 0.1$ & $-3.2 \pm 0.3$ \\
\hline Chlorolichens & $-0.7 \pm 0.0$ & $-1.1 \pm 0.3$ & $-1.6 \pm 0.3$ & $-2.0 \pm 0.4$ & $-2.4 \pm 0.5$ & $-2.9 \pm 0.6$ & $-3.7 \pm 0.6$ \\
\hline Moss-dominated crust & $-0.6 \pm 0.2$ & $-1.2 \pm 0.3$ & $-1.5 \pm 0.2$ & $-1.9 \pm 0.3$ & $-2.4 \pm 0.3$ & $-2.9 \pm 0.4$ & $-3.6 \pm 0.3$ \\
\hline Moss stems & $-0.4 \pm 0.2$ & $-0.6 \pm 0.3$ & $-0.8 \pm 0.5$ & $-0.7 \pm 0.2$ & $-0.9 \pm 0.3$ & $-1.2 \pm 0.4$ & $-1.7 \pm 0.2$ \\
\hline \multicolumn{8}{|l|}{ NP/DR ratio } \\
\hline Cyanobacteria/cyanolichen- dominated crust & $0.8 \pm 0.2$ & $0.7 \pm 0.3$ & $1.4 \pm 0.5$ & $1.2 \pm 0.2$ & $1.4 \pm 0.1$ & $1.2 \pm 0.1$ & $1.2 \pm 0.1$ \\
\hline Cyanobacteria/cyanolichens & $0.8 \pm 0.3$ & $0.9 \pm 0.1$ & $1.7 \pm 0.3$ & $1.1 \pm 0.2$ & $1.3 \pm 0.2$ & $1.4 \pm 0.5$ & $1.0 \pm 0.2$ \\
\hline Chlorolichen-dominated crust & $3.0 \pm 0.3$ & $3.0 \pm 0.3$ & $2.2 \pm 0.3$ & $2.0 \pm 0.2$ & $1.3 \pm 0.1$ & $1.0 \pm 0.1$ & $0.5 \pm 0.0$ \\
\hline Chlorolichens & $3.7 \pm 0.5$ & $3.2 \pm 0.6$ & $2.4 \pm 0.4$ & $2.1 \pm 0.4$ & $1.5 \pm 0.2$ & $1.0 \pm 0.0$ & $0.4 \pm 0.0$ \\
\hline Moss-dominated crust & $3.6 \pm 1.3$ & $1.9 \pm 1.2$ & $1.5 \pm 0.4$ & $1.4 \pm 0.5$ & $1.0 \pm 0.0$ & $0.4 \pm 0.2$ & $0.3 \pm 0.4$ \\
\hline Moss stems & $6.3 \pm 1.6$ & $5.2 \pm 1.6$ & $4.8 \pm 1.7$ & $6.0 \pm 2.5$ & $4.5 \pm 1.9$ & $2.4 \pm 1.0$ & $1.6 \pm 0.7$ \\
\hline
\end{tabular}

Data represent light compensation points $\left[\mu \mathrm{mol} \mathrm{m} \mathrm{s}^{-1} \mathrm{PPFD}\right]$, saturating irradiances $\left[\mu \mathrm{mol} \mathrm{m}^{-2} \mathrm{~s}^{-1}\right.$ PPFD], maximum NP rates [ $\mu \mathrm{mol}$ $\left.\mathrm{CO}_{2} \mathrm{~m}^{-2} \mathrm{~s}^{-1}\right]$, DR values at maximum NP $\left[\mu \mathrm{mol} \mathrm{CO} \mathrm{CO}^{-2} \mathrm{~s}^{-1}\right]$, and NP/DR ratios at $7,12,17,22,27,32$, and $37^{\circ} \mathrm{C}$. Measurements were performed at light intensities between 0 and $1500 \mu \mathrm{mol} \mathrm{m}^{-2} \mathrm{~s}^{-1}$ PPFD and $380 \mathrm{ppm} \mathrm{CO}_{2}$. Mean values $\pm \mathrm{SD}(\mathrm{n}=3)$ reflect physiological properties under optimum water conditions. Data on moss-dominated biocrusts and moss stems are taken from Weber et al. (2012)

reached significantly higher values $\left(4.0 \mu \mathrm{mol} \mathrm{CO}_{2}\right.$ $\mathrm{m}^{-2} \mathrm{~s}^{-1}$; Fig. 3, Table 2, Table S4) than the complete moss biocrust. Maximum NP at optimum temperature differed between chlorolichen- and cyanobacteria/ cyanolichen-dominated biocrusts (Fig. 3, Table 2, Table S4). 

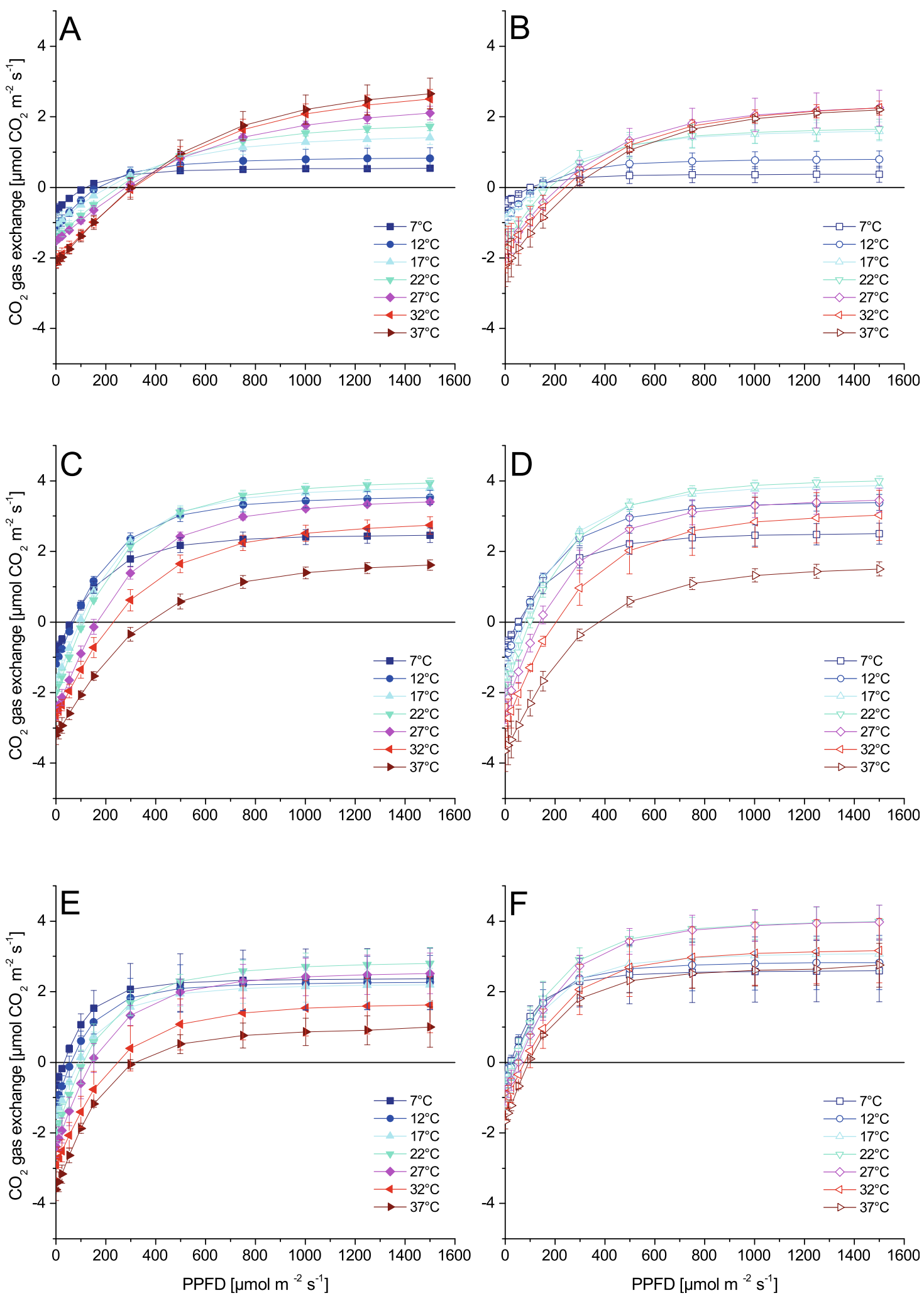
4Fig. 3 Response of $\mathrm{CO}_{2}$ gas exchange to increasing photosynthetic photon flux density (PPFD) at different temperatures of different biocrust types (closed symbols) and their isolated photoautotrophs (open symbols; $n=3$ ). (a) cyanobacteria/ cyanolichen-dominated biocrust; (b) isolated cyanobacteria/ cyanolichens; (c) chlorolichen-dominated biocrust; (d) isolated chlorolichens; (e) moss-dominated biocrust; (f) isolated moss stems. Measurements were conducted under optimum water conditions and at $380 \mathrm{ppm} \mathrm{CO}_{2}$. At the measured light intensities mean values $\pm \mathrm{SD}$ are shown. Smoothing of values was accomplished with the Smith function (Smith 1937, 1938) as described by Lange et al. (1991). Data on moss-dominated biocrusts and moss stems are taken from Weber et al. (2012)

For all types of biocrusts and the corresponding photoautotrophic components respiration rates increased with rising temperatures (Fig. 3; Table 2). Respiration rates of cyanobacteria/cyanolichen- and chlorolichen-dominated biocrusts did not differ significantly from these of their isolated photoautotrophs, but DR rates at maximum NP of chlorolichen-dominated biocrusts showed a larger increase in response to higher temperatures than the cyanobacteria/cyanolichendominated crusts (Table 2, Table S3). DR rates of moss-dominated biocrusts tended to be higher compared to other biocrusts types (Table 2, Table S3) and displayed a strong temperature-dependent increase, similar to chlorolichen-dominated biocrusts and their photoautotrophs (Table 2). In contrast to that, isolated moss stems had significantly lower DR rates $(-0.4$ to $-1.7 \mu \mathrm{mol} \mathrm{CO} \mathrm{C}^{-2} \mathrm{~s}^{-1}$; Table 2, Table S3).

$\mathrm{CO}_{2}$ gas exchange patterns in response to varying water, light and temperature values showed clear differences for the three types of biocrusts (Fig. 4). While $\mathrm{CO}_{2}$ gas exchange rates of cyanobacteria/cyanolichendominated biocrusts increased with rising temperatures and reached their maximum at $37^{\circ} \mathrm{C}$, chlorolichen- and moss-dominated biocrusts reached maximum NP values at $22(-27)^{\circ} \mathrm{C}$, which decreased again at higher temperatures (Table 2). Saturating light intensities of mossdominated biocrusts covered a wide range (468$1135 \mu \mathrm{mol} \mathrm{m} \mathrm{m}^{-2} \mathrm{~s}^{-1}$ PPFD); this was narrower for cyanobacteria/cyanolichen- and chlorolichendominated biocrusts (752-1301 and 723$1249 \mu \mathrm{mol} \mathrm{m} \mathrm{m}^{-2} \mathrm{~s}^{-1}$ PPFD, respectively; Fig. 4, Table 2). Mean NP/DR ratios (carbon use efficiency) reached $0.8-1.4$ for cyanobacteria/cyanolichen-dominated, 0.5-3.0 for chlorolichen-dominated, and 0.3-3.6 for moss-dominated biocrusts, with significant differences between chlorolichen- and moss-dominated biocrusts (two-way ANOVA; factors: biocrust type, temperature; Table 2, Table S4). These ratios decreased with rising temperatures in chlorolichen- and mossdominated biocrusts; in contrast, cyanobacteria/ cyanolichen-dominated biocrusts showed no clear pattern. Comparing complete biocrusts with their photoautotrophs, only moss-dominated biocrusts showed significant differences compared to moss stems, where values decreased along temperatures representing carbon use efficiency values from 6.3-1.6 (Table 2, Table S4). For the isolated photoautotrophs, NP/DR ratios differed significantly between moss stems and the other two types (Table 2, Table S4).

$\mathrm{CO}_{2}$ gas exchange rates measured in the field were in a similar range but had larger variability than the lab measurements. Both in the field and the lab, chlorolichen-dominated biocrusts reached the highest values, followed by moss- and cyanobacteria/ cyanolichen-dominated biocrusts (Fig. 5). Field- and lab measurements of cyanobacteria/cyanolichen- and chlorolichen-dominated biocrusts showed no significant differences with regard to maximum NP, whereas lab measurements of moss-dominated biocrusts were significantly higher than those measured in the field (DF = $5 ; \mathrm{t}$-value $=3.88 ; p$-value $=0.017 ;$ Fig. 5 ; Table S5).

\section{Discussion}

$\mathrm{CO}_{2}$ gas exchange properties differed substantially between biocrust types (Table S2, S3). Cyanobacteria/ cyanolichen-dominated biocrusts reached their highest $\mathrm{NP}$ rates at $37{ }^{\circ} \mathrm{C}$ with only moderate respiration rates, and were characterized by highest light compensation points and saturating light intensities. In comparison, chlorolichen-dominated crusts reached significantly higher maximum NP rates already at $22{ }^{\circ} \mathrm{C}$ (Table 2 , Table S4), whereas light compensation and saturating light intensities were somewhat lower (Table 2). Mossdominated biocrusts, which had already been measured in an earlier study, also had their maximum NP at $22{ }^{\circ} \mathrm{C}$, revealed large respiration rates at temperatures above $22{ }^{\circ} \mathrm{C}$ and had the lowest light compensation and saturation values, particularly at low ambient temperatures (Table 2). Properties of isolated photoautotrophs were similar to complete biocrusts for cyanobacteria/ cyanolichen- and chlorolichen-dominated biocrusts, 
Cyanobacteria/cyanolichendominated biocrust
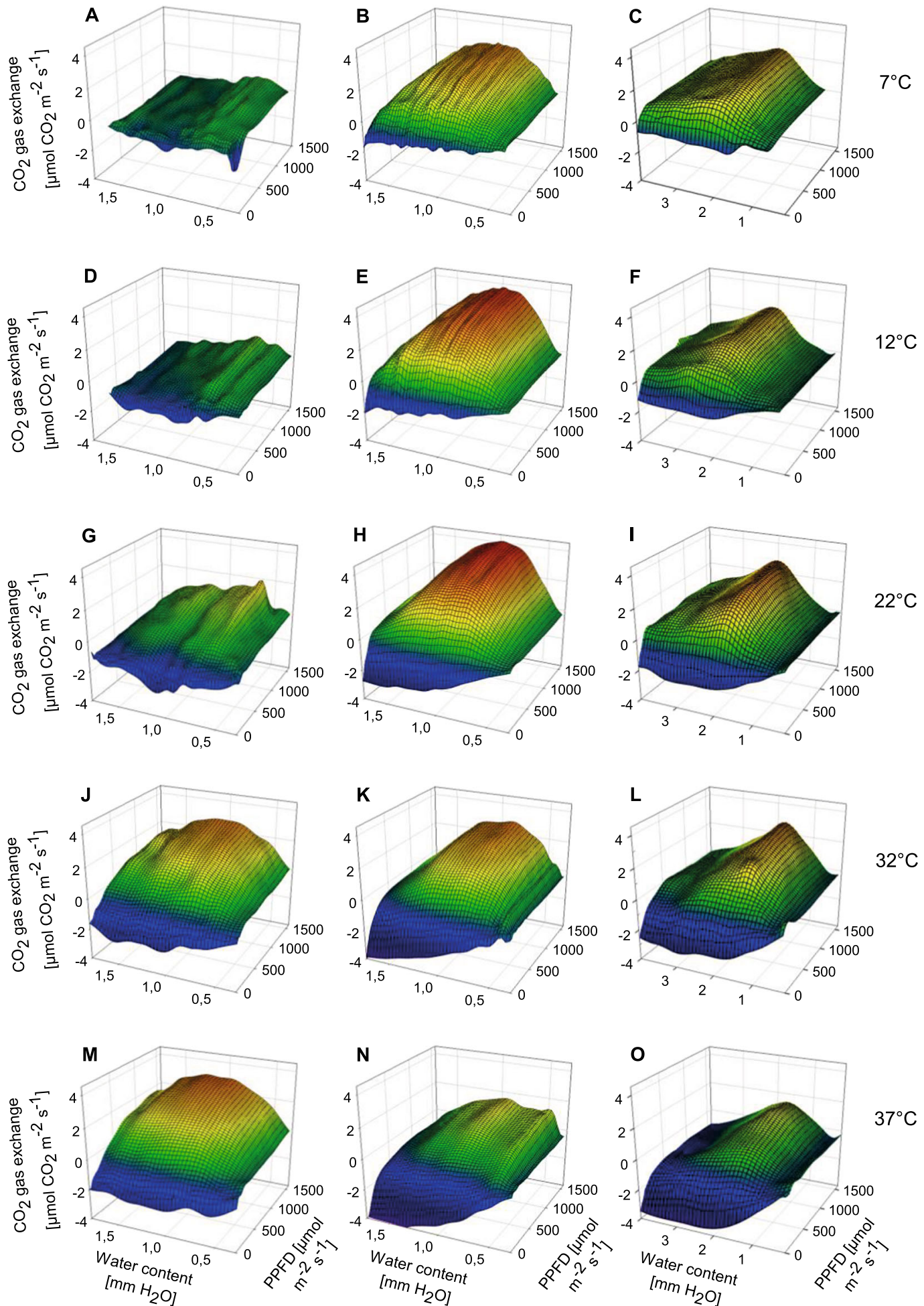
Fig. $4 \mathrm{CO}_{2}$ gas exchange of cyanobacteria/cyanolichen$(\mathbf{a}, \mathbf{d}, \mathbf{g}, \mathbf{j}, \mathbf{m})$, chlorolichen- $(\mathbf{b}, \mathbf{e}, \mathbf{h}, \mathbf{k}, \mathbf{n})$, and moss-dominated biocrusts $(\mathbf{c}, \mathbf{f}, \mathbf{i}, \mathbf{l}, \mathbf{0})$ at varying photosynthetic photon flux density (PPFD) and water content. Measurements at 7 (a-c), 12 (d-f), 22 $(\mathbf{g}-\mathbf{i}), 32(\mathbf{j}-\mathbf{l})$, and $37^{\circ} \mathrm{C}(\mathbf{m}-\mathbf{o})$, conducted at $380 \mathrm{ppm} \mathrm{CO}_{2}$, are shown. Data on moss-dominated biocrusts and moss stems are taken from Weber et al. (2012). Representative data of one replicate are shown

whereas isolated moss stems as compared to mossdominated biocrusts showed significantly higher maximum NP and significantly lower DR values with increasing differences towards high temperatures (Fig. 3, Table 2, Table S3).

Response of $\mathrm{CO}_{2}$ gas exchange to changing water contents

All biocrust types showed uniform optimum response curves of NP to water content, which were lower at low and high water contents (Fig. 2; Table 1). Low NP values at very low water contents are known to be caused by decreased photosynthetic activity due to dehydration and inhibition of the biochemical machinery (Lange et al. 1999). NP reduction at high water contents is a common phenomenon observed in many lichens, caused by increased diffusion resistance, which limits $\mathrm{CO}_{2}$ supply to the photobionts (Cowan et al. 1992; Green et al. 1994; Lange et al. 1997, 1999). At high water contents, a film of water within the lichen thallus may act as a barrier to $\mathrm{CO}_{2}$ diffusion, since its diffusion rate in water is $\sim 10^{4}$ times slower than in air (Green et al. 1981).

The current study region is characterized by semiarid to arid climate with precipitation being mostly restricted to minor rainfall events (Haarmeyer et al. 2010). Thus, an adaptation towards water suprasaturation does not seem to be necessary within this environment. This is different within other environments, as e.g., under temperate habitat conditions, where lichens like Cladonia convoluta $(=C$. foliacea) show no depression at high water contents (Lange and Green 2003, Green and Proctor 2016). On the other hand, NP rates of chlorolichen-dominated biocrusts from the Sonoran Desert were reduced to $\sim 60 \%$ of maximum rates after moderate suprasaturation (Büdel et al. 2013), and also in the cyanolichen Collema tenax, which forms a regular component in biocrusts of the southwestern US, depression of NP at high water contents has previously been observed (Lange et al. 1998). In mosses, depression of NP at high water contents is often less pronounced, as they have the greater ability to

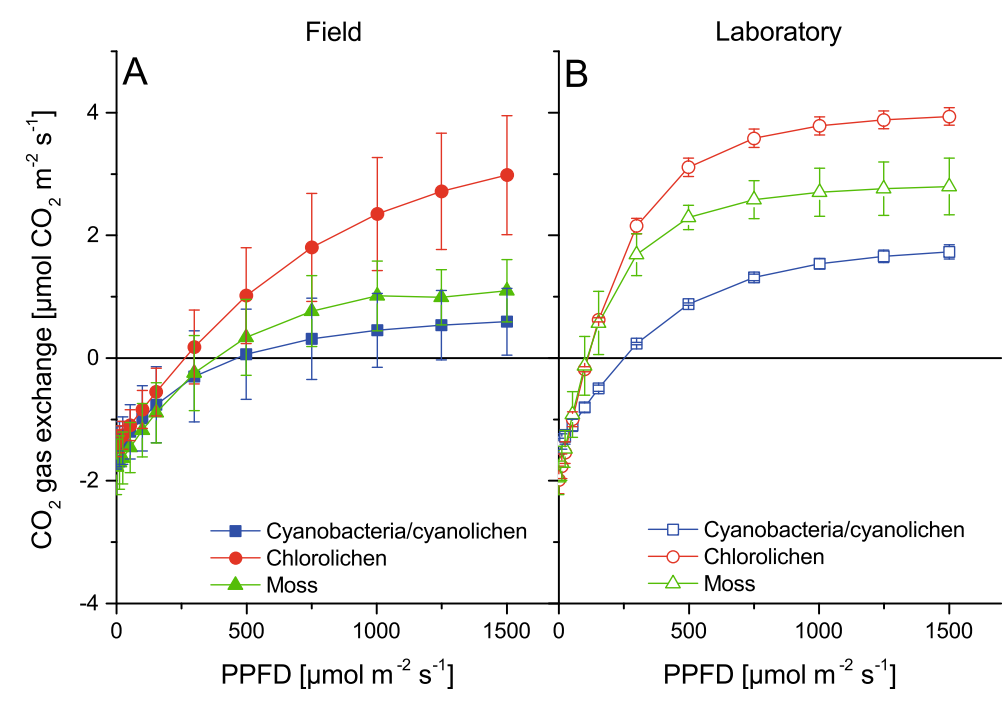

Fig. $5 \mathrm{CO}_{2}$ gas exchange rates at increasing photosynthetic photon flux density (PPFD), $22{ }^{\circ} \mathrm{C}, 380 \mathrm{ppm}$, and optimum water content obtained during field (A; closed symbols) and lab measurements (B; open symbols). Different colors represent values of cyanobacteria/cyanolichen- (blue square), chlorolichen- (red circle), and moss-dominated biocrusts (green triangle). Smoothing of values was accomplished with the Smith function (Smith 1937,
1938) as described in Lange et al. (1991). Data on moss-dominated biocrusts and moss stems are taken from Weber et al. (2012). Mean values \pm SD are shown at measured light intensity steps (lab measurements $\mathrm{n}=3$; field measurements: cyanobacteria/ cyanolichen- and chlorolichen-dominated biocrusts $n=5$, mossdominated biocrusts $n=6$ ) 
separate stored extracellular water from photosynthetic exchange surfaces (Green and Proctor 2016). Bryophytes adapt to high water contents with shoots and ornamentation of their surfaces to provide capillary spaces for water storage, while they also have unstructured surfaces, which serve for gas exchange without $\mathrm{CO}_{2}$ diffusion barriers (Green et al. 1994; Green and Proctor 2016).

These physiological characteristics with regard to water utilization are reflected by different microhabitats utilized by the investigated biocrust types. Mosses and liverworts have been shown to preferably grow under shrubs and in shade-protected exposure (Bowker et al. 2016; Li et al. 2016), where water conditions are more favorable (Weber et al. 2012), resulting in reduced soil evaporation and thus longer crust activity on the microscale. This can be an important factor in determining crustal thickness and distribution patterns (Veste and Littmann 2006). On the contrary, the cover of lichens, with the most frequent species being Collema coccophorum, Peccania arabica, Psora crenata and Placidium squamulosum, is very patchy due to the large spatial variability of soils with regard to $\mathrm{pH}$, electrical conductivity, cover of stones and anthropogenic impacts (Haarmeyer et al. 2010). In the current study, the optimum water contents of cyanobacteria/cyanolichendominated biocrusts ranged between 0.52 and $0.78 \mathrm{~mm} \mathrm{H}_{2} \mathrm{O}$, being similar to these of cyanolichendominated biocrusts from the Sonoran Desert, where values reached $0.72-1.17 \mathrm{~mm}$ (Petula richardsii) and 0.48-1.05 $\mathrm{mm} \mathrm{H}_{2} \mathrm{O}$ (Petula patellata). The cyanolichen Collema tenax reached maximum NP values at slightly higher water contents of $1.06 \pm 0.12 \mathrm{~mm} \mathrm{H}_{2} \mathrm{O}$ (Lange et al. 1998; Büdel et al. 2013). Biocrusts of the Boodjamulla National Park (northwestern Queensland), mainly consisting of the cyanobacteria Sypmplocastrum purpurascens and Scytonema sp. with small amounts of Nostoc sp. reached optimum NP values at very similar water contents of $0.49-0.77 \mathrm{~mm}$ $\mathrm{H}_{2} \mathrm{O}$ (Büdel et al. 2018).

Chlorolichen-dominated biocrusts accomplished maximum NP values at water contents ranging between 0.75 and $1.15 \mathrm{~mm} \mathrm{H}_{2} \mathrm{O}$, whereas isolated photoautotrophs needed $0.34-0.73 \mathrm{~mm} \mathrm{H}_{2} \mathrm{O}$ for optimum NP rates. Lange et al. (1997) calculated very similar optimum water contents (between 0.39 and $0.94 \mathrm{~mm} \mathrm{H}_{2} \mathrm{O}$ ) for the chlorolichens Diploschistes diacapsis, Psora cerebriformis, and Squamarina lentigera. Optimum water contents of moss-dominated biocrusts were very high, ranging from 1.76 to $2.38 \mathrm{~mm} \mathrm{H}_{2} \mathrm{O}$, and nearly similar to the values determined for isolated moss stems (1.77 to $2.28 \mathrm{~mm} \mathrm{H}_{2} \mathrm{O}$ ). High optimum water contents of moss-dominated biocrusts have also been observed in previous studies (Dilks and Proctor 1979) and are reasonable, as bryophytes are known to have substantial water storage capacities (Warren 2003; Belnap 2006; Seitz et al. 2017).

The standardized precipitation-evaporation index (SPEI), taking into account both precipitation and potential evapotranspiration (PET) in determining drought, predicts a general increase in drought cover for South Africa during the next decades (Vicente-Serrano et al. 2015; Department of Environmental Affairs 2017). Thus, our data indicate that mosses with their high water demands may suffer most severely from the anticipated climate change, being followed by chlorolichendominated biocrusts, while cyanobacteria-dominated biocrusts with their low water-demands and high temperature optimum seem to be well-adapted to changing climatic conditions. These results confirm previous experimental studies, where bryophytes were shown to be killed by smaller more frequent precipitation events (Reed et al. 2012).

Water compensation points of cyanobacteria/ cyanolichen- $\left(0.36 \pm 0.04 \mathrm{~mm} \mathrm{H}_{2} \mathrm{O}\right)$ and chlorolichendominated biocrusts $\left(0.29 \pm 0.08 \mathrm{~mm} \mathrm{H}_{2} \mathrm{O}\right)$ tended to be slightly, but insignificantly, lower than those of mossdominated biocrusts $\left(0.72 \pm 0.32 \mathrm{~mm} \mathrm{H}_{2} \mathrm{O}\right.$; Table 1 , Table S2). This is in line with previous observations, where lichen-dominated biocrusts (Diploschistes diacapsis, Psora cerebriformis, and Squamarina lentigera) have been described to have low water compensation points between 0.05 and $0.27 \mathrm{~mm} \mathrm{H}_{2} \mathrm{O}$ (Lange et al. 1997).

Average maximum NP rates of water response curves at $17{ }^{\circ} \mathrm{C}$ and $500 \mu \mathrm{mol} \mathrm{m} \mathrm{m}^{-2}$ PPFD showed no significant differences between measurements in the beginning and in the end of the experiments for cyanobacteria/cyanolichen- and chlorolichendominated biocrusts as well as their photoautotrophs, indicating that the organisms did not suffer from the long measuring period.

DR of cyanobacteria/cyanolichen-dominated biocrusts and photoautotrophs in response to varying water content differed from other biocrust types, as values were strongly depressed at high water contents. Such a depression of DR upon supersaturation was also observed for Collema tenax as described by Lange et al. 
(1998). For lichen-dominated biocrusts with Diploschistes diacapsis, Psora cerebriformis, and Squamarina lentigera as dominating photoautotrophs also previous studies showed no depression of DR at high water contents (Lange et al. 1997).

Response of $\mathrm{CO}_{2}$ gas exchange to changing light and temperature

$\mathrm{CO}_{2}$ gas exchange measurements revealed that the NP of cyanobacteria/cyanolichen-dominated biocrusts and their photoautotrophs was adapted to high temperatures $\left(\sim 27-37^{\circ} \mathrm{C}\right)$, whereas the temperature optimum of chlorolichen- and moss-dominated biocrusts and the photoautotrophs ranged around $22-27^{\circ} \mathrm{C}$. These measurements are in line with earlier studies by Lange et al. (1998), who observed a temperature optimum above $30{ }^{\circ} \mathrm{C}$ for Collema tenax, whereas that of the chlorolichens Psora cerebriformis and Diploschistes diacapsis was around $20-25^{\circ} \mathrm{C}$. They pointed out that cyanobacterial photobionts might contribute to the high temperature optimum of cyanolichens, as an adaptation to high temperatures has also been observed for cyanobacterial epilithic dryland lichens (i.e. Petula capensis; Wessels and Kappen 1993) and the tropical lichen Dictyonema glabratum (Green et al. 1994). The photobiont of $C$. tenax is protected against photoinhibition by a dark thallus, which allows the utilization of high light intensities when the sun is also warming the lichen (Lange et al. 1998). Our maximum measured temperature was $37^{\circ} \mathrm{C}$, and we thus could not determine if that is the optimum temperature for cyanobacteria/cyanolichen-dominated biocrusts or if it is even somewhat higher. However, Lange et al. (1998) investigated Collema tenax at temperatures up to $41{ }^{\circ} \mathrm{C}$ and they found NP rates strongly decreased at temperatures above $37^{\circ} \mathrm{C}$, thus supporting a temperature optimum at $\sim 37^{\circ} \mathrm{C}$. Zhao et al. (2010) presented an optimum temperature range between 20 and $25^{\circ} \mathrm{C}$ for mossand between 25 and $30^{\circ} \mathrm{C}$ for cyanobacteria-dominated biocrusts of the Hilly Loess Plateau region of China. At low temperatures of 7 or $12{ }^{\circ} \mathrm{C}$ cyanobacteria/ cyanolichen-dominated biocrusts and their photoautotrophs showed by far lower maximum NP rates ( 0.4 to $0.8 \mu \mathrm{mol} \mathrm{CO}_{2} \mathrm{~m}^{-2} \mathrm{~s}^{-1}$ ) compared to moss- and chlorolichen-dominated biocrusts and their photoautotrophs $\left(2.3-3.5 \mu \mathrm{mol} \mathrm{CO} \mathrm{m}^{-2} \mathrm{~s}^{-1}\right)$.

Maximum NP values of cyanobacteria/cyanolichenand moss-dominated biocrusts were similar with 2.7 and
$2.8 \mu \mathrm{mol} \mathrm{CO}_{2} \mathrm{~m}^{-2} \mathrm{~s}^{-1}$, respectively, whereas chlorolichen-dominated crusts reached significantly higher values of $3.9 \mu \mathrm{mol} \mathrm{CO} \mathrm{CO}^{-2} \mathrm{~s}^{-1}$ (Table 2; Table S4). The values of cyanobacteria/cyanolichendominated biocrusts are similar to the results of lab measurements by Brostoff et al. (2005) for cyanobacteria-dominated crusts growing on dunes $\left(\sim 2.5 \mu \mathrm{mol} \mathrm{CO} \mathrm{m}^{-2} \mathrm{~s}^{-1}\right)$. Cyanolichen-dominated crusts with Petula richardsii and Petula patellata had somewhat lower maximum NP rates of 1.4 and $1.9 \mu \mathrm{mol} \mathrm{CO}$ $\mathrm{m}^{-2} \mathrm{~s}^{-1}$, respectively (Büdel et al. 2013). Only Collema tenax from Utah, USA, reached much higher maximum $\mathrm{NP}$ values of about $5.3 \pm 0.9 \mu \mathrm{mol} \mathrm{CO} \mathrm{C}^{-2} \mathrm{~s}^{-1}$ under optimum conditions (Lange et al. 1998). This may be reinforced by the cyanolichen-dominance in these samples, whereas our samples comprised only a minor fraction of cyanolichens but were dominated by cyanobacteria, which generally tend to have lower NP rates (Lange 2003a). High maximum NP rates of chlorolichen-dominated biocrusts and lichens isolated from the soil have also been observed for Psora cerebriformis, Diploschistes diacapsis, and Squamarina lentigera, which reached maximum NP values between 2.6 and $5.2 \mu \mathrm{mol} \mathrm{CO} \mathrm{m}^{-2} \mathrm{~s}^{-1}$ (Lange et al. 1997). Mossdominated biocrusts reached maximum NP rates similar to measurements of Pleurozium schreberi $(\sim 2 \mu \mathrm{mol} \mathrm{CO} 2$ $\mathrm{m}^{-2} \mathrm{~s}^{-1}$; Williams and Flanagan 1996) and higher than those obtained for temperate rainforest mosses $(\sim 1.3$ to $1.8 \mu \mathrm{mol} \mathrm{CO} \mathrm{Cm}^{-2} \mathrm{~s}^{-1}$; DeLucia et al. 2003).

Maximum NP rates of isolated photoautotrophs of cyanobacteria/cyanolichen- and chlorolichendominated biocrusts at optimum temperatures were similar to the complete biocrusts $\left(2.3\right.$ and $4.0 \mu \mathrm{mol} \mathrm{CO}_{2}$ $\mathrm{m}^{-2} \mathrm{~s}^{-1}$, respectively), whereas isolated moss stems reached by far higher values $\left(4.0 \mu \mathrm{mol} \mathrm{CO} \mathrm{CO}_{2} \mathrm{~m}^{-2}\right)$ (Table 2, Table S4). This illustrates that the heterotrophic components of moss-dominated biocrusts have high respiration rates, whereas the heterotrophs in cyanobacteria/cyanolichen- and chlorolichendominated biocrusts had only a minor effect. Several studies provide evidence that different stages of biocrusts control the microbial community composition and this may affect physiological properties such as soil respiration (Cleveland et al. 2007; Liu et al. 2017, Maier et al. 2018). One has to keep in mind, however, that also the photoautotrophic components isolated from the underlying substrate were not sterile, but certainly comprised some bacteria and fungi on their surface and potentially also within thalli (Maier et al. 2014). The 
high respiration rates of moss-dominated biocrusts in this study are likely caused by large quantities of dead organic matter (i.e., plant litter and other dead moss material), which may feed larger quantities of bacteria and fungi compared to other biocrust types (Cleveland et al. 2007). Particularly high respiration rates of mossdominated biocrusts from northern China were also observed by Zhao et al. (2014) after major rainfall events. In studies conducted in the central part of the Iberian Peninsula soil respiration rates were twice as high in well-developed late compared to early successional biocrusts and they were significantly higher in areas dominated by biocrusts compared to open microsites with low biocrust cover (Castillo-Monroy et al. 2011; Escolar et al. 2015). Thus, lower soil respiration rates of cyanobacteria/cyanolichen- and chlorolichen-dominated biocrusts may also partly be explained by lower nutrient availability within the interspaces between shrubs.

Carbon use efficiency (NP/DR) of chlorolichen- and moss-dominated biocrusts was in the commonlyobserved range of $\sim 0.3-3.6$, which has also been observed for other biocrust lichens (Lange et al. 1997). For biocrusts of a temperate site in Germany and of Antarctica, carbon use efficiencies of $2-5$ were observed (Colesie et al. 2014; Gypser et al. 2016). Values of cyanobacteria/cyanolichen-dominated biocrusts, on the other hand, were surprisingly low (0.7-1.4), as the cyanolichen Collema tenax previously was shown to have a quotient of $\sim 0.5-5$ (Lange et al. 1998). Carbon use efficiency of isolated moss stems was highest, ranging between 1.6 and 6.3 with highest values reached at low temperatures. Similarly high or even higher quotients of $\sim 8-10$ have also been previously described for the epilithic lichen Lecanora muralis (Lange 2003b).

Summarizing the factorial analyses, cyanobacteria/ cyanolichen-dominated biocrusts reached the highest NP values under relatively low moisture and high temperature conditions, chlorolichen-dominated biocrusts under low moisture but medium temperature levels, and moss-dominated biocrusts under high moisture and medium temperature regimes. Similar physiological properties were also shown for cyanobacteria- and moss-dominated biocrusts of Loess Plateau region, China (Zhao et al. 2010).

Although biocrusts in desert regions are only active during a minor fraction of the year, as their activity is inevitably linked to moisture availability, they may still play a relevant role in carbon cycling of drylands, where vascular plant vegetation is sparse (Sancho et al. 2016). It was recently shown that their metabolic activity can be predicted from relative air humidity and air temperature within the study area (Raggio et al. 2017). Thus, knowledge of these environmental conditions and the physiological properties of biocrusts are indispensable for calculations of ecological performance and carbon balance (Lange et al. 1997).

Based on their physiological performance, biocrusts not only affect global biogeochemical cycles (Weber et al. 2015; Porada et al. 2017), but the different biocrust types have also been shown to host different microbial communities (Maier et al. 2018). Soil texture also varies between biocrust types, as cyanobacteria-dominated biocrusts have a layering structure of fine and coarse substrate fractions, which does not exist in mossdominated biocrusts (Garcia-Pichel et al. 2016). Cyanobacteria, lichens, and mosses have been shown to accelerate weathering of minerals, and biocrusts of later successional stages, which are more active and complex, were suggested to more strongly accelerate mineral weathering than earlier successional ones (Chen et al. 2009). Additionally, biocrust soils have been found to be enriched in $\mathrm{C}$ and $\mathrm{N}$, whereas they were depleted in $\mathrm{Ca}, \mathrm{Cr}, \mathrm{Mn}, \mathrm{Cu}, \mathrm{Zn}$, As, and $\mathrm{Zr}$ contents (Beraldi-Campesi et al. 2009).

\section{Respiration of heterotrophic biocrust fraction}

Soil respiration was only clearly visible in $\mathrm{CO}_{2}$ gas exchange measurements of moss-dominated biocrusts and moss stems as previously described by Weber et al. (2012). The respiration rates of the heterotrophic fraction without moss stems were up to $-2.0 \mu \mathrm{mol} \mathrm{CO}_{2}$ $\mathrm{m}^{-2} \mathrm{~s}^{-1}$, which caused by far lower absolute values of NP/DR ratios of moss-dominated biocrusts as compared to moss stems (Table 2, Table S3). We could not find such high soil respiration rates in the other biocrust types, as they did not reveal big differences in $\mathrm{CO}_{2}$ gas exchange values between complete biocrusts and photoautotrophic components (Figs. 3 and 4; Table 2). Soil respiration could be caused by abiotic $\mathrm{CO}_{2}$ formation from soil carbonates as well as by the biological activity of heterotrophic organisms within the soil (Rey 2015; Sancho et al. 2016). Weber et al. (2012) already underlined the importance of measuring complete biocrusts as well as the isolated components of them.

Soil moisture can also be relevant, as moisture pulses have been shown to cause enhanced soil respiration, 
which may last for weeks or even longer (Bowling et al. 2011). Recent studies also indicate that varying microbial communities may cause different soil respiration rates of different types of biocrusts (Li et al. 2013; Liu et al. 2017; Chilton et al. 2017; Maier et al. 2018).

Field vs. laboratory work

Our measurements revealed that field and lab measurements of cyanobacteria/cyanolichen- and chlorolichendominated biocrusts corresponded well, illustrating that factorial analyses conducted in the lab are well suited to determine the physiological properties of biocrusts under field conditions (Fig. 5). The results show that our sample treatment was suitable and had no significant effect on biocrust vitality. Only in formerly analyzed moss-dominated biocrusts there was a difference in rates between the lab and the field, with lab rates significantly higher (Fig. 5, Table S5). This was likely caused by problems with overall vitality of the samples, which required the introduction of a correction factor (for further details see Weber et al. 2012).

Our study allowed us to fulfill our three main research aims. Highest maximum NP rates were reached by chlorolichen-dominated biocrusts $\left(3.9 \mu \mathrm{mol} \mathrm{CO}_{2}\right.$ $\mathrm{m}^{-2} \mathrm{~s}^{-1}$ ), followed by cyanobacteria/cyanolichen- and moss-dominated biocrusts, which reached similar values (2.7 and $2.8 \mu \mathrm{mol} \mathrm{CO}_{2} \mathrm{~m}^{-2} \mathrm{~s}^{-1}$, respectively). Cyanobacteria/cyanolichen-dominated biocrusts were adapted to high temperatures $\left(\sim 37^{\circ} \mathrm{C}\right)$, whereas the temperature optimum of chlorolichen- and mossdominated biocrusts ranged around $22{ }^{\circ} \mathrm{C}$. All biocrust types showed optimum curves with regard to water. Both light compensation and light saturation points increased with increasing temperatures and were highest for cyanobacteria/cyanolichen-dominated biocrusts, being followed by chlorolichen- and moss-dominated biocrusts. Respiration of the heterotrophic biocrust fraction was only clearly visible for moss-dominated biocrusts, whereas for cyanobacteria/cyanolichen- and chlorolichen-dominated biocrusts it was negligible.

Conclusions

The physiological activity of all three biocrust types was primarily driven by water availability, being influenced by both light and temperature conditions. Cyanobacteria-dominated biocrusts reached optimum NP rates at lowest water contents and highest light intensities and temperatures. Chlorolichen-dominated biocrusts took a medium position, as optimum NP was measured at medium water contents and light intensities and temperatures of $22{ }^{\circ} \mathrm{C}$. Moss-dominated biocrusts needed largest amounts of water, lowest light intensities and also medium temperatures for optimum NP rates. These differential physiological adaptations are likely reflected by differences in carbon allocation patterns over the course of the year and moss-dominated biocrusts are anticipated to suffer most from the expected changes of climate.

Acknowledgements Open access funding provided by Max Planck Society. This work was supported by the German Research Foundation (Project numbers WE 2393/2-1, WE 2393/2-2) and the Max Planck Society. Research in South Africa was conducted with South African research permits (No. 22/2008 and 38/2009) and the appendant export permits. We would like to thank Burkhard Büdel and Ulrich Pöschl for their overall support and the provision of lab space, Jayne Belnap for her very helpful comments on the manuscript, and Tobias Graf and Matthias Bass for measurement of moss-dominated biocrusts and their photoautotrophs, which have been published in a previous publication. BW would like to thank Paul Crutzen for awarding her a Nobel Laureate fellowship.

\section{Compliance with ethical standards}

Conflict of interest The authors declare no conflict of interest.

Open Access This article is distributed under the terms of the Creative Commons Attribution 4.0 International License (http:// creativecommons.org/licenses/by/4.0/), which permits unrestricted use, distribution, and reproduction in any medium, provided you give appropriate credit to the original author(s) and the source, provide a link to the Creative Commons license, and indicate if changes were made.

\section{References}

Arnon DI, McSwain BD, Tsujimoto HY, Wada K (1974) Photochemical activity and components of membrane preparations from blue-green algae. I. Coexistence of two photosystems in relation to chlorophyll a and removal of phycocyanin. Biochim Biophys Acta-Bioenerg 357:231-245

Belnap J (2006) The potential roles of biological soil crusts in dryland hydrologic cycles. Hydrol Process 20:3159-3178

Belnap J, Harper KT (1995) Influence of cryptobiotic soil crusts on elemental content of tissue of two desert seed plants. Arid Soil Res Rehabil 9:107-115

Belnap J, Rosentreter R, Kaltenecker J, et al (2001) Biological soil crusts: ecology and management. Technical reference 1730 
2. Bureau of Land Management, United States Department of the Interior

Belnap J, Weber B, Büdel B (2016) Biological soil crusts as an organizing principle in drylands. In: Weber B, Büdel B, Belnap J (eds) Biological soil crusts: an organizing principle in drylands, Ecological Studies 226. Springer International Publishing, pp 3-13

Beraldi-Campesi H, Hartnett HE, Anbar AD, Gordon GW, GarciaPichel F (2009) Effects of biological soil crusts on soil elemental concentrations: implications for biogeochemistry and as traceable biosignatures of ancient life on land. Geobiology 7:348-359

Beyschlag W, Wittland M, Jentsch A, Steinlein T (2008) Soil crusts and disturbance benefit plant germination, establishment and growth on nutrient deficient sand. Basic Appl Ecol 9:243-252

Bowker MA, Belnap J, Büdel B, Sannier C, Pietrasiak N, Eldridge DJ, Rivera-Aguilar V (2016) Controls on distribution patterns of biological soil crusts at micro- to global scales. In: Weber B, Büdel B, Belnap J (eds) Biological soil crusts: an organizing principle in drylands, Ecological Studies 226. Springer International Publishing, pp 173-197

Bowling DR, Grote EE, Belnap J (2011) Rain pulse response of soil $\mathrm{CO}_{2}$ exchange by biological soil crusts and grasslands of the semiarid Colorado plateau, United States. J Geophys Res Biogeosci 116(3):1-17

Brostoff WN, Sharifi MR, Rundel PW (2005) Photosynthesis of cryptobiotic soil crusts in a seasonally inundated system of pans and dunes in the western Mojave Desert, CA: field studies. Flora 200:592-600

Büdel B, Darienko T, Deutschewitz K, Friedl T, Mohr KI, Salisch M, Reisser W, Weber B (2009) Southern african biological soil crusts are ubiquitous and highly diverse in drylands, being restricted by rainfall frequency. Microb Ecol 57:229247

Büdel B, Vivas M, Lange OL (2013) Lichen species dominance and the resulting photosynthetic behavior of Sonoran Desert soil crust types (Baja California, Mexico). Ecol Process 2:6

Büdel B, Williams WJ, Reichenberger H (2018) Annual net primary productivity of a cyanobacteria dominated biological soil crust in the Gulf savanna, Queensland, Australia. Biogeosciences 15:491-505

Caesar J, Tamm A, Ruckteschler N, Weber B (2017) Revisiting chlorophyll extraction methods in biological soil crusts methodology for determination of chlorophyll a and chlorophyll $\mathrm{a}+\mathrm{b}$ as compared to previous methods. Biogeosciences Discuss. https://doi.org/10.5194/bg-2017-396

Caesar J, Tamm A, Ruckteschler N, Weber B (2018) Revisiting chlorophyll extraction methods in biological soil crusts methodology for determination of chlorophyll a and chlorophyll $\mathrm{a}+\mathrm{b}$ as compared to previous methods. Biogeosciences 15:1415-1424

Campbell SE, Seeler J, Golubic S (1989) Desert crust formation and soil stabilization. Arid Soil Res Rehabil 3:217-228

Castillo-Monroy AP, Maestre FT, Rey A, Soliveres S, GarcíaPalacios P (2011) Biological soil crust microsites are the main contributor to soil respiration in a semiarid ecosystem. Ecosystems 14:835-847

Chen R, Zhang Y, Li Y, Wei W, Zhang J, Wu N (2009) The variation of morphological features and mineralogical components of biological soil crusts in the Gurbantunggut Desert of northwestern China. Environ Geol 57:1135-1114

Chilton AM, Eldridge DJ, Neilan BA (2017) Biocrust morphology is linked to marked differences in microbial community composition. Plant Soil. https://doi.org/10.1007/s11104017-3442-3

Cleveland CC, Nemergut DR, Schmidt SK, Townsend AR (2007) Increases in soil respiration following labile carbon additions linked to rapid shifts in soil microbial community composition. Biogeochemistry 82:229-240

Coe KK, Belnap J, Sparks JP (2012) Precipitation-driven carbon balance controls survivorship of desert biocrust mosses. Ecology 93:1626-1636

Colesie C, Green TGA, Haferkamp I, Büdel B (2014) Habitat stress initiates changes in composition, $\mathrm{CO}_{2}$ gas exchange and $\mathrm{C}$-allocation as life traits in biological soil crusts. ISME $8: 2104-2115$

Colesie C, Williams L, Büdel B (2017) Water relations in the soil crust lichen Psora decipiens are optimized via anatomical variability. Lichenologist 49(5):483-492

Cowan IR, Lange OL, Green TGA (1992) Carbon-dioxide exchange in lichens: determination of transport and carboxylation characteristics. Planta 187:282-294

DeLucia EH, Turnbull MH, Walcroft AS, Griffin KL, Tissue DT, Glenny D, McSeveny TM, Whitehead D (2003) The contribution of bryophytes to the carbon exchange for a temperate rainforest. Glob Chang Biol 9:1158-1170

Department of Environmental Affairs (2017) South Africa's 2nd Annual Climate Change Report. Pretoria, Department of Environmental Affairs

Dilks T, Proctor M (1979) Photosynthesis respiration and water content in bryophytes. New Phytol 80:97-114

Dojani S, Büdel B, Deutschewitz K, Weber B (2011) Rapid succession of biological soil crusts after experimental disturbance in the succulent Karoo, South Africa. Appl Soil Ecol 48:263-269

Dojani S, Kauff F, Weber B, Büdel B (2014) Genotypic and phenotypic diversity of cyanobacteria in biological soil crusts of the succulent Karoo and Nama Karoo of southern Africa. Microb Ecol 67(2):286-301

Elbert W, Weber B, Burrows S, Steinkamp J, Büdel B, Andreae MO, Pöschl U (2012) Contribution of cryptogamic covers to the global cycles of carbon and nitrogen. Nat Geosci 5:459 462

Eldridge D, Ferris JM (1999) Recovery of populations of the soil lichen Psora crenata after disturbance in arid South Australia. Rangel J 21(2):194-198

Escolar C, Maestre FT, Rey A (2015) Biocrusts modulate warming and rainfall exclusion effects on soil respiration in a semi-arid grassland. Soil Biol Biochem 80:9-17

Frahm J-P, Schumm F, Stapper NJ (2010) Epiphytische Flechten als Umweltgütezeiger: - eine Bestimmungshilfe. Books on Demand, Norderstedt, Germany, pp 1-164

Garcia-Pichel F, Felde VJMNL, Drahorad SL, Weber B (2016) Microstructure and weathering processes within biological soil crusts. In: Weber B, Belnap J, Büdel B (eds) Biological soil crusts: an organizing principle in drylands, ecological studies 226. Springer International Publishing, Switzerland, pp 237-255

Green TGA, Proctor MCF (2016) Physiology of photosynthetic organisms within biological soil crusts: their adaptation, 
flexibility, and plasticity. In: Weber B, Büdel B, Belnap J (eds) Biological soil crusts: an organizing principle in drylands, Ecological Studies 226. Springer International Publishing, pp 347-381

Green TGA, Snelgar WP, Brown DH (1981) Carbon dioxide exchange in lichens. Carbon dioxide exchange through the cyphellate lower cortex of Sticta latifrons Rich. New Phytol 88:421-426

Green TGA, Lange OL, Cowan IR (1994) Ecophysiology of lichen photosynthesis, the role of water status and thallus diffusion resistances. Cryptogam Bot 4:166-178

Gypser S, Herppich WB, Fischer T, Lange P, Veste M (2016) Photosynthetic characteristics and their spatial variance on biological soil crust covering initial soils of post-mining sites in lower Lusatia. Flora 220:103-116

Haarmeyer D H, Luther-Mosebach J, Dengler J, Schmiedel U, Finckh M, Berger K, Deckert J, Domptail SE, Dreber N, Gibreel T, Grohmann C, Gröngröft A, Haensler A, Hanke W, Hoffmann A, Husted LB, Kangombe FN, Keil M, Krug CB, Labitzky T, Linke T, Mager D, Mey W, Muche G, Naumann C, Pellowski M, Powrie LW, Pröpper M, Rutherford MC, Schneiderat U, Strohbach BJ, Vohland K, Weber B, Wesuls D, Wisch U, Zedda L, Büdel B, Darienko T, Deutschewitz K, Dojani S, Erb E, Falk T, Friedl T, Kanzler SE, Limpricht C, Linsenmair KE, Mohr K, Oliver T, Petersen A, Rambold G, Zeller U, Austermühle R, Bausch J, Bösing BM, Classen N, Dorendorf J, Dorigo W, Esler KJ, Etzold S, Graiff A, Grotehusmann L, Hecht J, Hoyer P, Kongor RY, Lang H, Lieckfeld LAB, Oldeland J, Peters J, Röwer IU, September ZM, Sop TK, van Rooyen MW, Weber J, Willer J, Jürgens N (2010) The BIOTA Observatories. In: Jürgens N, Haarmeyer DH, Luther-Mosebach J, Dengler J, Finckh M, Schmiedel U (eds) Biodiversity in southern Africa. Volume 1: Patterns at local scale - the BIOTA Observatories. Klaus Hess Publishers, Göttingen \& Windhoek. pp 6-801.

Kunz NS, Hoffman MT, Weber B (2012) Effects of heuweltjies and utilization on vegetation patterns in the succulent Karoo, South Africa. J Arid Environ 87:198-205

Lange OL (1980) Moisture content and $\mathrm{CO}_{2}$ exchange of lichens I. Influence of temperature on moisture-dependent net photosynthesis and dark respiration in Ramalina maciformis. Oecologia 45:82-87

Lange OL (2003a) Photosynthesis of soil-crust biota as dependent on environmental factors. In: Belnap J, Lange OL (eds) Biological soil crusts: structure, function, and management. Springer Berlin Heidelberg, Berlin, Heidelberg, pp 217-240

Lange OL (2003b) Photosynthetic productivity of the epilithic lichen Lecanora muralis: long-term field monitoring of $\mathrm{CO}_{2}$ exchange and its physiological interpretation: I dependence of photosynthesis on water content, light, temperature, and $\mathrm{CO}_{2}$ concentration from laboratory measurements. Flora 197:233-249

Lange OL (2003c) Photosynthetic productivity of the epilithic lichen Lecanora muralis: long-term field monitoring of $\mathrm{CO}_{2}$ exchange and its physiological interpretation: III diel, seasonal and annual carbon budgets. Flora 198:277-292

Lange OL, Green TGA (2003) Photosynthetic performance of a foliose lichen of biological soil crust communities: long-term monitoring of the $\mathrm{CO}_{2}$ exchange of Cladonia convoluta under temperate habitat conditions. Biblio Lichenol 86: 257-280
Lange OL, Meyer A, Ullmann I, Zellner H (1991) Mikroklima, Wassergehalt und Photosynthese von Flechten in der küstennahen Nebelzone der Namib-Wüste: Messungen während der herbstlichen Witterungsperiode. Flora 185: 233-266

Lange OL, Belnap J, Reichenberger H, Meyer A (1997) Photosynthesis of green algal soil crust lichens from arid lands in southern Utah, USA: role of water content on light and temperature responses of $\mathrm{CO}_{2}$ exchange. Flora 192:1-15

Lange OL, Belnap J, Reichenberger H (1998) Photosynthesis of the cyanobacterial soil-crust lichen Collema tenax from arid lands in southern Utah, USA: role of water content on light and temperature responses of $\mathrm{CO}_{2}$ exchange. Funct Ecol 12: 195-202

Lange OL, Green TGA, Reichenberger H (1999) The response of lichen photosynthesis to external $\mathrm{CO}_{2}$ concentration and its interaction with thallus water-status. J Plant Physiol 154: $157-166$

Leavitt S, Westberg M, Sohrabi M, Elix JA, Timdal E, Nelsen MP, St. Clair LL, Williams L, Wedin M, Lumbsch T (2018) Multiple, distinct intercontinental lineages but isolation of Australian populations in a cosmopolitan lichen-forming fungal taxon, Psora decipiens (Psoraceae, Ascomycota). Front Microbiol 9. https://doi.org/10.3389/fmicb.2018.00283

Lenhart K, Weber B, Elbert W, Steinkamp J, Clough T, Crutzen P, Pöschl U, Keppler F (2015) Nitrous oxide and methane emissions from cryptogamic covers. Glob Chang Biol 21: 3889-3900

Li X-R, Jia X-H, Long L-Q, Zerbe S (2005) Effects of biological soil crusts on seed bank, germination and establishment of two annual plant species in the Tengger Desert (N China). Plant Soil 277:375-385

Li H, Colica G, Wu PP, Li D, Rossi F, De Philippis R, Liu Y (2013) Shifting species interaction in soil microbial community and its influence on ecosystem functions modulating. Microb Ecol 65(3):700-708

Li B, Gao J, Wang X, Ma L, Cui Q, Veste M (2016) Effects of biological soil crusts on water infiltration and evaporation Yanchi Ningxia, Maowusu Desert, China. Int J Sediment Res 31(4):311-323

Liu L, Liu Y, Zhang P, Song G, Hui R, Wang Z, Wang J (2017) Development of bacterial communities in biological soil crusts along a revegetation chronosequence in the Tengger Desert, Northwest China. Biogeosciences 14:3801-3814

Maier S, Schmidt TSB, Zheng L, Peer T, Wagner V, Grube M (2014) Analyses of dryland biological soil crusts highlight lichens as an important regulator of microbial communities. Biodivers Conserv 23:1735-1755

Maier S, Muggia L, Kuske CR, Grube M (2016) Bacteria and nonlichenized fungi within biological soil crusts. In: Weber B, Büdel B, Belnap J (eds) Biological soil crusts: an organizing principle in drylands. Springer International Publishing, pp $81-100$

Maier S, Tamm A, Wu D, Caesar J, Grube M, Weber B (2018) Photoautotrophic organisms control microbial abundance, diversity, and physiology in biological soil crusts. ISME. https://doi.org/10.1038/s41396-018-0062-8

Meusel H, Tamm A, Kuhn U et al (2018) Emission of nitrous acid from soil and biological soil crusts represents a dominant source of HONO in the remote atmosphere in Cyprus. Atmos Chem Phys 18:799-813 
Porada P, Weber B, Elbert W, Pöschl U, Kleidon A (2014) Estimating impacts of lichens and bryophytes on global biogeochemical cycles. Global Biogeochem Cy 28:71-85

Porada P, Pöschl U, Kleidon A, Beer C, Weber B (2017) Estimating global nitrous oxide emissions by lichens and bryophytes with a process-based productivity model. Biogeosciences 14:1593-1602

Raggio J, Allan Green TG, Sancho LG, Pintado A, Colesie C, Weber B, Büdel B (2017) Metabolic activity duration can be effectively predicted from macroclimatic data for biological soil crust habitats across Europe. Geoderma 306:10-17

Reed SC, Coe KK, Sparks JP, Housman DC, Zelikova TJ, Belnap J (2012) Changes to dryland rainfall result in rapid moss mortality and altered soil fertility. Nat Clim Chang 2:752755

Rey A (2015) Mind the gap: non-biological processes contributing to soil $\mathrm{CO}_{2}$ efflux. Glob Chang Biol 21:1752-1761

Rodríguez-Caballero E, Paul M, Tamm A, Caesar J, Büdel B, Escribano P, Hill J, Weber B (2017) Biomass assessment of microbial surface communities by means of hyperspectral remote sensing data. Sci Total Environ 15(586):1287-1297

Rodríguez-Caballero E, Belnap J, Büdel B, Crutzen P, Andreae MO, Pöschl U, Weber B (2018) Dryland photoautotrophic soil surface communities endangered by global change. Nat Geosci 11:185-189

Ronen R, Galun M (1984) Pigment extraction from lichens with dimethyl sulfoxide (DMSO) and estimation of chlorophyll degradation. Environ Exp Bot 24:239-245

Rosentreter R, Bowker M, Belnap J (2007) A field guide to biological soil crusts of Western U.S. Drylands - common lichens and bryophytes. US Government Printing Office, Denver, CO, pp 1-104

Sancho LG, Belnap J, Colesie C, Raggio J, Weber B (2016) Carbon budgets of biological soil crusts at micro-, meso-, and global Scales. In: Weber B, Büdel B, Belnap J (eds) Biological soil crusts: an organizing principle in drylands, Ecological Studies 226. Springer International Publishing, pp 287-304

Seitz S, Nebel M, Goebes P, Goebes P, Käppeler K, Schmidt K, Shi X, Song Z, Webber CL, Weber B, Scholten T (2017) Bryophyte-dominated biological soil crusts mitigate soil erosion in an early successional Chinese subtropical forest. Biogeosciences 14:5775-5788

Seppelt RD, Downing AJ, Deane-Coe KK, Zhang Y, Zhang J (2016) Bryophytes within biological soil crusts. In: Weber B, Büdel B, Belnap J (eds) Biological soil crusts: an organizing principle in drylands, Ecological Studies 226. Springer International Publishing, pp 101-120

Smith EL (1937) The influence of light and carbon dioxide on photosynthesis. J Gen Physiol 20:807-830

Smith EL (1938) Limiting factors in photosynthesis: light and carbon dioxide. J Gen Physiol 22:21-35
Van Jaarsveld E (1987) The succulent riches of South Africa and Namibia. Aloe 24:45-92

Veste M, Littmann T (2006) Dewfall and its geo-ecological implication for biological surface crusts in desert sand dunes (north-western Negev, Israel). J Arid Land Stud 16(3):139147

Vicente-Serrano, Sergio M. \& National Center for Atmospheric Research Staff (Eds). Last modified 18 Jul 2015. "The climate data guide: standardized precipitation evapotranspiration index (SPEI)." Retrieved from https://climatedataguide. ucar.edu/climate-data/standardized-precipitationevapotranspiration-index-spei

Warren SD (2003) Synopsis: influence of biological soil crusts on arid land hydrology and soil stability. In: Belnap J, Lange OL (eds) Biological soil crusts: structure, function, and management. Springer Berlin Heidelberg, Berlin, Heidelberg, pp 349-360

Weber B, Graf T, Bass M (2012) Ecophysiological analysis of moss-dominated biological soil crusts and their separate components from the succulent Karoo, South Africa. Planta 236:129-139

Weber B, Wessels DC, Deutschewitz K, Dojani S, Reichenberger H, Büdel B (2013) Ecological characterization of soilinhabiting and hypolithic soil crusts within the Knersvlakte, South Africa. Ecol Process 2:8

Weber B, Wu D, Tamm A, Ruckteschler N, Rodríguez-Caballero E, Steinkamp J, Meusel H, Elbert W, Behrendt T, Sörgel M, Cheng Y, Crutzen PJ, Su H, Pöschl U (2015) Biological soil crusts accelerate the nitrogen cycle through large NO and HONO emissions in drylands. P Natl Acad Sci USA 112: 15384-15389

Wessels D, Kappen L (1993) Photosynthetic performance of rockcolonising lichens in the mountain Zebra National Park, South Africa. Koedoe 36(1):27-48

Williams TG, Flanagan LB (1996) Effect of changes in water content on photosynthesis, transpiration and discrimination against ${ }^{13} \mathrm{CO}_{2}$ and $\mathrm{C}^{18} \mathrm{O}^{16} \mathrm{O}$ in Pleurozium and Sphagnum. Oecologia 108:38-46

Williams L, Colesie C, Ullmann A, Westberg M, Wedin M, Büdel B (2017) Lichen acclimation to changing environments: photobiont switching vs. climate-specific uniqueness in Psora decipiens. Ecol Evol 7:2560-2574

Zhao Y, Xu M, Belnap J (2010) Response of biocrusts' photosynthesis to environmental factors: a possible explanation of the spatial distribution of biocrusts in the hilly loess plateau region of China. Acta Ecol Sin 30:4668-4675

Zhao Y, Qin N, Weber B, Xu M (2014) Response of biological soil crusts to raindrop erosivity and underlying influences in the hilly loess plateau region, China. Biodivers Conserv 23: 1669-1686 